\title{
A Model for the Coupled Disease Dynamics of HIV and HSV-2 with Mixing Among and Between Genders
}

\author{
Christina Alvey ${ }^{\mathrm{a}, *}$, Zhilan Feng $^{\mathrm{a}}$, John Glasser ${ }^{\mathrm{b}}$ \\ ${ }^{a}$ Department of Mathematics, Purdue University, 150 N. University Street, West Lafayette, IN 47907, USA \\ ${ }^{b}$ Population Biology, Ecology and Evolution, Emory University, 1462 Clifton Road, Atlanta, GA 30322, USA
}

\begin{abstract}
Evidence indicates that those with genital herpes (HSV-2) infections have greater risks of infection by HIV; and, once co-infected, are more likely to transmit HIV. To better understand the interactions between HIV and HSV-2, we construct a mathematical model that describes the joint dynamics. A new feature of this model is the inclusion of both heterosexual and homosexual interactions. We derive and interpret the basic and invasion reproduction numbers for HIV and HSV-2 using the approach of next-generation matrices. We then perform scenario analyses and conduct a sensitivity analysis to investigate the impact of the model parameters on the reproduction numbers and disease prevalences. We conclude that homosexual transmission drastically changes the disease prevalences; hence, it is important to account for this interaction as models that ignore homosexuality may greatly underestimate the disease burden.
\end{abstract}

Keywords: HIV, HSV-2, population dynamics, basic reproduction number, invasion reproduction number, sensitivity analysis

\section{Introduction}

Human immunodeficiency virus (HIV) and herpes simplex virus type-2 (HSV-2) are sexually transmitted diseases that affect thousands of individuals in the United States. HIV is a virus that targets cells of the immune system, with about 50, 000 new cases in the United States every year and roughly $16 \%$ unaware of their infection[1]. The Centers for Disease Control and Prevention (CDC) report that about one in six Americans have HSV-2; however, many infected individuals may be unaware of their infections due to a lack of symptoms[2]. Individuals with HSV-2 experience a lifelong infection, alternating between periods of acute HSV-2 in which they are infectious and periods of latent HSV-2 in which the virus remains dormant. While both diseases have treatments available to reduce viral shedding or to prevent reactivation, currently neither is curable.

Further, HSV-2 may facilitate the spread of HIV[1, 2]. Evidence suggests that HSV-2 infection increases the risk of acquiring HIV. This is mainly due to the lesions that are caused by HSV-2 infection and facilitate infection

\footnotetext{
${ }^{*}$ Corresponding author

Email addresses: clorenzo@purdue.edu (Christina Alvey), fengz@purdue.edu (Zhilan Feng), jwglass@emory .edu (John Glasser)
}

(C) 2015. This manuscript version is made available under the Elsevier user license 
by other STDs, making infected individuals 2 to 5 times more likely to contract HIV [1, 2, 3, 4]. Co-infection also allows for an enhanced transmission of HIV as co-infected individuals have a higher concentration of HIV in their genital fluids than individuals with HIV only[1, 3, 4]. Moreover, between $40-80 \%$ of individuals diagnosed with HIV are co-infected with HSV-2, with a large portion of these individuals from among the homosexual male population[5, 6, 7].

Many mathematical models discuss HIV or HSV-2 alone [8, 9, 10, 11, 12], and a few discuss the relationship between HIV and HSV-2 [5, 6, 13, 14]. For instance, Blower and Ma [6] coupled an HIV compartmental model with an HSV-2 model to investigate the effects of HSV-2 prevalence on the number of new cases of HIV; Abu-Raddad et al.[5] consider a homosexual male population and suggest that HSV-2 prevalence, if near an endemic level, may predict the spread of HIV; and Foss et al.[13] developed a dynamic model to estimate the HIV infections directly due to HSV-2 in a heterosexual population. Most disease models incorporate either the heterosexual population alone [11, 13, 14] or the homosexual population alone [5, 6, 9]. However, with the growing number of same-sex couples in the United States and other countries, which may be due to the growing acceptance of homosexuality, it is important to consider these types of interactions[15]. In Malunguza et al.[12], an HIV model is presented that separates the male population into heterosexual and homosexual, with the possibility of interaction to create a bisexual class. The female population is considered to be completely heterosexual and the model only considers HIV transmission. Including the possibility of homosexual relations was beneficial in that Malunguza et al. were able to consider various treatment scenarios corresponding to the different types of interactions. To our knowledge, a model that incorporates heterosexual and homosexual encounters among both male and female populations and includes the role of co-infection has not yet been considered.

Our model is based on the model of Feng et al., in which an ODE model is introduced that describes the transmission dynamics of HIV and HSV-2 to examine the role of co-infection and investigate possible treatment strategies[14]. The population is divided by gender and includes risk groups among the female population, with transmission occurring only through heterosexual contact[14]. In contrast, our paper takes into account transmission through either heterosexual or homosexual behavior and investigates the impact of these sexual encounters on the coupled dynamics of HIV and HSV-2. To focus on the complexity of these new interactions between genders, we first consider a model that ignores risk groups. The main goals of our paper include determining the role of the preference for heterosexual versus homosexual contact on the reproduction numbers and disease prevalences and investigating the effect of co-infection of HIV and HSV-2 on efforts to reduce the HIV epidemic.

We organize the paper as follows: In Section 2, we present the model. We derive and explain the basic reproduction numbers for HIV and HSV-2 in Section 3 . Section 4 presents the invasion reproduction numbers for both diseases. Results from numerical simulations of the model are illustrated in Section 5. A sensitivity analysis is also included in Section 5. Finally, in Section 6, we discuss the results of our model. 


\section{Model Formulation}

Here we present a model that describes the transmission dynamics of HIV and HSV-2 in a population with both heterosexual and homosexual encounters. We consider two population groups based on gender, males (denoted by a subscript $m$ ) and females $(f)$, with transmission occurring only due to sexual engagements. We incorporate sexual partnerships between genders as well as partnerships within each gender. Because gender mixing complicates the model, we ignore the heterogeneity in age-of-infection and use ordinary differential equations to describe the transmission dynamics of both HIV and HSV-2.

The population is divided into six classes per gender at time $t$ : susceptible $\left(S_{i}(t)\right)$, HIV-positive $\left(H_{i}(t)\right)$, individuals in the acute stage of HSV-2 $\left(A_{i}(t)\right)$, individuals in the latent stage of HSV-2 $\left(L_{i}(t)\right)$, co-infected individuals in the acute stage of HSV-2 $\left(P_{i}(t)\right)$, and co-infected individuals in the latent stage of HSV-2 $\left(Q_{i}(t)\right)$, $i=m, f$. Denote the total population of group $i$ as $N_{i}=S_{i}+H_{i}+A_{i}+L_{i}+P_{i}+Q_{i}$ at each time $t, i=m, f$. It is important to note that the population under consideration is the sexually active population. Hence, entry into the system $\left(\Lambda_{i}\right)$ represents an individual becoming sexually active; and exit from the system $\left(\mu_{i}^{S}, \mu_{i}^{H}, \mu_{i}^{A}, \mu_{i}^{L}, \mu_{i}^{P}\right.$, and $\mu_{i}^{Q}$ ) represents cessation of sexual activity, which may or may not be due to death. Moreover, we do not consider AIDS in our model, assuming that those individuals with AIDS refrain from sexual activity. Thus, acquisition of AIDS is another means of exiting our system. The strength of HIV and HSV-2 infections are represented by $\lambda_{i, j}^{H}$ and $\lambda_{i, j}^{V}, i=m, f, j=m, f$, respectively (see [2.3). As we would like to consider both heterosexual and homosexual relations, we define $q_{i}$ to be the fraction of partnerships that an $i$ individual has with another $i$ individual, $i=m, f$. We also consider enhancement terms $\delta_{i}^{H}, \delta_{i}^{A}$, and $\delta_{i}^{L}$, which account for the greater susceptibility of individuals with one disease to the other. For instance, we may assume that individuals with acute HSV-2 are more likely to obtain HIV $\left(\delta_{i}^{A}\right)$. We allow for the possibility of co-infection increasing the infectivity of a disease with the terms $\sigma_{i}^{H}$ and $\sigma_{i}^{V}$. For example, if an individual has both diseases, he may be more infectious with HIV than an individual who only has HIV $\left(\sigma_{i}^{H}\right)$. In addition, we include progression from the acute stage of HSV-2 to the latent stage $\left(\omega_{i}^{A}, \omega_{i}^{P}\right)$, and reactivation from the latent stage to the acute stage $\left(\gamma_{i}^{L}, \gamma_{i}^{Q}\right)$. The system is presented in 2.1] with an explanation of variables and parameters in Table 1 and a visual representation of the model in Figure 1

$$
\left\{\begin{array}{l}
\dot{S}_{i}=\Lambda_{i}-\left(\lambda_{i, i}^{H}+\lambda_{j, i}^{H}+\lambda_{i, i}^{V}+\lambda_{j, i}^{V}\right) S_{i}-\mu_{i}^{S} S_{i} \\
\dot{H}_{i}=\left(\lambda_{i, i}^{H}+\lambda_{j, i}^{H}\right) S_{i}-\delta_{i}^{H}\left(\lambda_{i, i}^{V}+\lambda_{j, i}^{V}\right) H_{i}-\mu_{i}^{H} H_{i} \\
\dot{A}_{i}=\left(\lambda_{i, i}^{V}+\lambda_{j, i}^{V}\right) S_{i}-\delta_{i}^{A}\left(\lambda_{i, i}^{H}+\lambda_{j, i}^{H}\right) A_{i}+\gamma_{i}^{L} L_{i}-\left(\mu_{i}^{A}+\omega_{i}^{A}\right) A_{i} \\
\dot{L}_{i}=\omega_{i}^{A} A_{i}-\delta_{i}^{L}\left(\lambda_{i, i}^{H}+\lambda_{j, i}^{H}\right) L_{i}-\left(\mu_{i}^{L}+\gamma_{i}^{L}\right) L_{i} \\
\dot{P}_{i}=\delta_{i}^{H}\left(\lambda_{i, i}^{V}+\lambda_{j, i}^{V}\right) H_{i}+\delta_{i}^{A}\left(\lambda_{i, i}^{H}+\lambda_{j, i}^{H}\right) A_{i}+\gamma_{i}^{Q} Q_{i}-\left(\mu_{i}^{P}+\omega_{i}^{P}\right) P_{i} \\
\dot{Q}_{i}=\delta_{i}^{L}\left(\lambda_{i, i}^{H}+\lambda_{j, i}^{H}\right) L_{i}+\omega_{i}^{P} P_{i}-\left(\mu_{i}^{Q}+\gamma_{i}^{Q}\right) Q_{i} \\
i=m, f, \quad j=m, f, \quad j \neq i .
\end{array}\right.
$$


Table 1: Description of Model Parameters

\begin{tabular}{|c|c|}
\hline Symbol & Description \\
\hline$m, f$ & male, female \\
\hline$N_{i}(t)$ & Total population of group $i$ at time $t$ \\
\hline$S_{i}(t)$ & Number of susceptible individuals of group $i$ at time $t$ \\
\hline$H_{i}(t)$ & Number of HIV-positive individuals of group $i$ at time $t$ \\
\hline$A_{i}(t)$ & Number of individuals in the acute stage of HSV-2 of group $i$ at time $t$ \\
\hline$L_{i}(t)$ & Number of individuals in the latent stage of HSV-2 of group $i$ at time $t$ \\
\hline$P_{i}(t)$ & Number of co-infected individuals in the acute stage of HSV-2 of group $i$ at time $t$ \\
\hline$Q_{i}(t)$ & Number of co-infected individuals in the latent stage of HSV-2 of group $i$ at time $t$ \\
\hline$\mu_{i}$ & Rate of exiting sexual activity for group $i$ \\
\hline$\Lambda_{i}$ & Total recruitment rate of group $i$ \\
\hline$q_{i}$ & Fraction of partnerships an individual of group $i$ has with another individual of group $i$ \\
\hline$\delta_{i}^{A}$ & Enhanced susceptibility of individuals with acute HSV-2 to HIV \\
\hline$\delta_{i}^{L}$ & Enhanced susceptibility of individuals with latent HSV-2 to HIV \\
\hline$\delta_{i}^{H}$ & Enhanced susceptibility of individuals with HIV to HSV-2 \\
\hline$\sigma_{i}^{V}$ & Enhanced infectivity of individuals with both STDs when transmitting HSV-2 \\
\hline$\sigma_{i}^{H}$ & Enhanced infectivity of individuals with both STDs when transmitting HIV \\
\hline$\omega_{i}^{A}, \omega_{i}^{P}$ & Rate of acute HSV-2 becoming latent \\
\hline$\gamma_{i}^{L}, \gamma_{i}^{Q}$ & Reactivation rate of latent HSV-2 \\
\hline$b_{i}$ & Rate of sexual contacts of individuals of group $i$ \\
\hline$\beta_{i, i}^{H}$ & $\begin{array}{l}\text { Probability per partner of HIV transmission by an HIV-positive individual of group } i \text { to a partner of } \\
\text { the same group }\end{array}$ \\
\hline$\beta_{i, j}^{H}$ & $\begin{array}{l}\text { Probability per partner of HIV transmission by an HIV-positive individual of group } i \text { to a partner of } \\
\text { group } j\end{array}$ \\
\hline$\beta_{i, i}^{V}$ & $\begin{array}{l}\text { Probability per partner of HSV-2 transmission by an HSV-2-positive individual of group } i \text { to a partner } \\
\text { of the same group }\end{array}$ \\
\hline$\beta_{i, j}^{V}$ & $\begin{array}{l}\text { Probability per partner of HSV-2 transmission by an HSV-2-positive individual of group } i \text { to a partner } \\
\text { of group } j\end{array}$ \\
\hline$\lambda_{i, i}^{H}(t)$ & Rate of infection of individuals of group $i$ by individuals of the same group with HIV at time $t$ \\
\hline$\lambda_{j, i}^{H}(t)$ & Rate of infection of individuals of group $i$ by individuals of group $j$ with HIV at time $t$ \\
\hline$\lambda_{i, i}^{V i}(t)$ & Rate of infection of individuals of group $i$ by individuals of the same group with HSV-2 at time $t$ \\
\hline$\lambda_{j, i}^{i, i}(t)$ & Rate of infection of individuals of group $i$ by individuals of group $j$ with HSV-2 at time $t$ \\
\hline
\end{tabular}

Let $b_{i}$ be the number of contacts that an individual of group $i$ has per unit time. Let $q_{m}$ be the fraction of contacts that a male individual has with another male individual. Similarly, let $q_{f}$ be the fraction of partnerships that a female individual has with another female. Note that males have the same number of contacts with females as females have with males. Thus we have the following balance equation:

$$
\left(1-q_{m}\right) b_{m} N_{m}=\left(1-q_{f}\right) b_{f} N_{f} \text {. }
$$

The forces of infection in 2.1) are derived as follows:

$$
\begin{aligned}
\lambda_{i, i}^{H} & =q_{i} b_{i} \beta_{i, i}^{H} \frac{H_{i}+\sigma_{i}^{H}\left(P_{i}+Q_{i}\right)}{N_{i}} \\
\lambda_{j, i}^{H} & =\left(1-q_{i}\right) b_{i} \beta_{j, i}^{H} \frac{H_{j}+\sigma_{j}^{H}\left(P_{j}+Q_{j}\right)}{N_{j}} \\
\lambda_{i, i}^{V} & =q_{i} b_{i} \beta_{i, i}^{V} \frac{A_{i}+\sigma_{i}^{V} P_{i}}{N_{i}} \\
\lambda_{j, i}^{V} & =\left(1-q_{i}\right) b_{i} \beta_{j, i}^{V} \frac{A_{j}+\sigma_{j}^{V} P_{j}}{N_{j}}, \quad i=m, f, \quad j=m, f, \quad j \neq i .
\end{aligned}
$$

Note that only individuals infected with acute HSV-2 may infect others with HSV-2, while co-infected individuals 


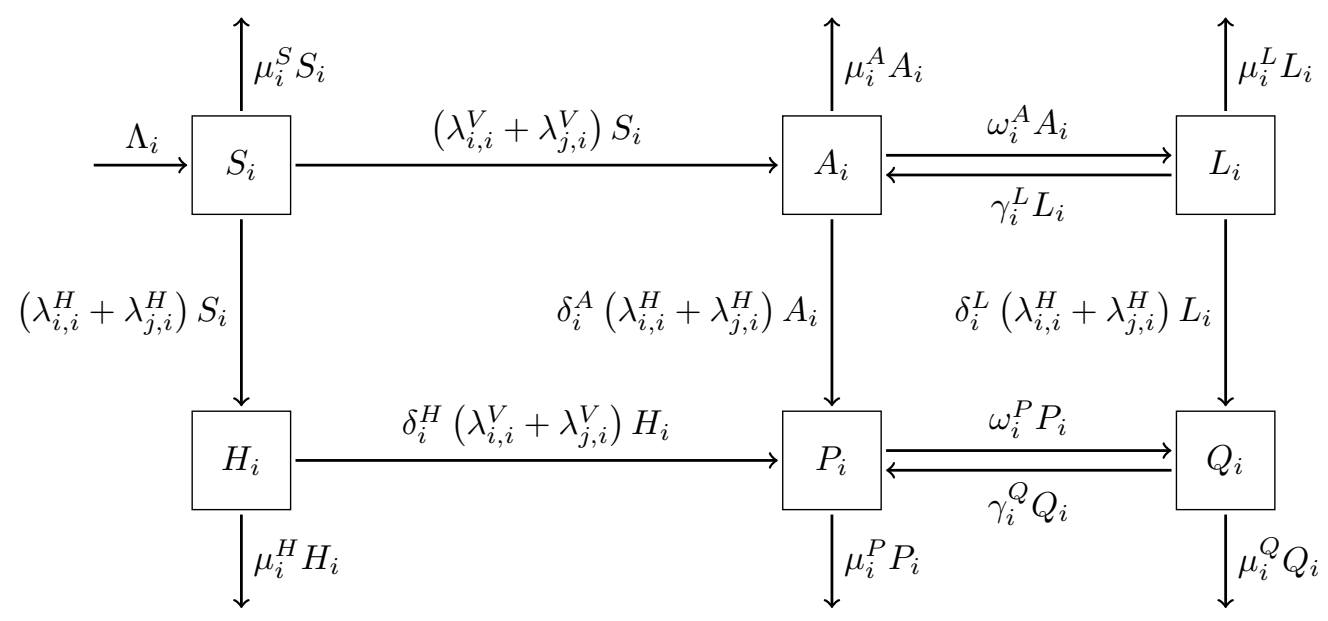

Figure 1: The compartmentalized model illustrating disease dynamics.

with acute or latent HSV-2 may infect others with HIV. Using the balance equation 2.2 , we may write:

$$
\begin{aligned}
\lambda_{j, i}^{H} & =\left(1-q_{j}\right) b_{j} \beta_{j, i}^{H} \frac{H_{j}+\sigma_{j}^{H}\left(P_{j}+Q_{j}\right)}{N_{i}} \\
\lambda_{j, i}^{V} & =\left(1-q_{j}\right) b_{j} \beta_{j, i}^{V} \frac{A_{j}+\sigma_{j}^{V} P_{j}}{N_{i}}, \quad i=m, f, \quad j=m, f, \quad j \neq i .
\end{aligned}
$$

\section{The Basic Reproduction Numbers}

Our first aim is to find the basic reproduction number of each disease, i.e. $R_{0}^{H}$ and $R_{0}^{V}$, around the disease-free equilibrium (DFE). The DFE is $\left(S_{i}^{0}:=\frac{\Lambda_{i}}{\mu_{i}^{S}}, 0,0,0,0,0\right), i=m, f$. Note that the basic reproduction number is the average number of secondary infections caused by one infected individual in a population that is completely susceptible to that pathogen and without the other. If this value is greater than one, infection will spread in the population; if this value is less than one, the infection will die out (i.e. the value one is a threshold).

\subsection{Basic Reproduction number for $H I V\left(R_{0}^{H}\right)$}

We first find the basic reproduction number for HIV (i.e. assuming that there is no HSV-2 in the population). The basic reproduction number is given by (see Appendix $\mathrm{A}$ for the derivation):

$$
R_{0}^{H}=\rho\left(F V^{-1}\right)=\frac{1}{2}\left(R_{f, f}^{H}+R_{m, m}^{H}+\sqrt{\left(R_{f, f}^{H}-R_{m, m}^{H}\right)^{2}+4 R_{f, m}^{H} R_{m, f}^{H}}\right),
$$

where

$$
\begin{aligned}
& R_{i, i}^{H}=\frac{q_{i} b_{i} \beta_{i, i}^{H}}{\mu_{i}^{H}} \\
& R_{i, j}^{H}=\frac{\left(1-q_{i}\right) b_{i} \beta_{i, j}^{H}}{\mu_{i}^{H}}, \quad i=m, f, \quad j=m, f, \quad j \neq i .
\end{aligned}
$$




\subsubsection{Case 1: Only heterosexual interactions.}

First consider the case where $q_{m}, q_{f}=0$, i.e. only heterosexual relations. Then (3.1) reduces to:

$$
R_{0}^{H}=\sqrt{R_{f, m}^{H} R_{m, f}^{H}}
$$

Note that $b_{f} \beta_{f, m}^{H}$ is the rate at which HIV-positive females infect males, while $\frac{1}{\mu_{f}^{H}}$ is the amount of time that a female stays in the $H_{f}$ class. Hence, $R_{f, m}^{H}=\frac{b_{f} \beta_{f, m}^{H}}{\mu_{f}^{H}}$ is the total number of males that one HIV-positive female will infect while sexually active. Similarly, $R_{m, f}^{H}=\frac{b_{m} \beta_{m, f}^{H}}{\mu_{m}^{H}}$ is the number of females that an HIV-positive male will infect in his sexual lifetime. Thus 3.2 is the square root of the average number of secondary infections caused by one HIV-positive person through heterosexual contact.

\subsubsection{Case 2: Only homosexual interactions.}

Next, consider the case where $q_{m}, q_{f}=1$, i.e. only homosexual relations. In this case, we have eigenvalues of $F V^{-1}: R_{f, f}^{H}=\frac{b_{f} \beta_{f, f}^{H}}{\mu_{f}^{H}}$ and $R_{m, m}^{H}=\frac{b_{m} \beta_{m, m}^{H}}{\mu_{m}^{H}}$. Note that $R_{f, f}^{H}$ is the reproduction number for the system excluding the male population and $R_{m, m}^{H}$ is the reproduction number for the system excluding the female population. We define:

$$
R_{0}^{H}=\max \left(R_{f, f}^{H}, R_{m, m}^{H}\right)
$$

The first term represents the total number of females that one HIV-positive female will infect within her sexual lifetime via only homosexual encounters. Similarly, the second term represents the total number of males one HIV-positive male individual will infect within his sexual lifetime via homosexual relationships.

\subsection{Basic Reproduction number for $H S V-2\left(R_{0}^{V}\right)$}

Next we will find the basic reproduction number for HSV-2 (i.e. assuming that there is no HIV in the population). In this case, the basic reproduction number is given by $\rho\left(F V^{-1}\right)$ (see Appendix $\mathrm{B}$ for the derivation):

$$
R_{0}^{V}=\frac{1}{2}\left(R_{f, f}^{V}+R_{m, m}^{V}+\sqrt{\left(R_{f, f}^{V}-R_{m, m}^{V}\right)^{2}+4 R_{f, m}^{V} R_{m, f}^{V}}\right)
$$

where

$$
\begin{aligned}
R_{i, i}^{V} & =q_{i} b_{i} \beta_{i, i}^{V} T_{i}^{A}, \\
R_{i, j}^{V} & =\left(1-q_{i}\right) b_{i} \beta_{i, j}^{V} T_{i}^{A}, \\
T_{i}^{A} & =\frac{\mu_{i}^{L}+\gamma_{i}^{L}}{\left(\mu_{i}^{A}+\omega_{i}^{A}\right)\left(\mu_{i}^{L}+\gamma_{i}^{L}\right)-\omega_{i}^{A} \gamma_{i}^{L}}, \quad i=m, f, \quad j=m, f, \quad j \neq i .
\end{aligned}
$$

The interpretation of $T_{i}^{A}$ can be found in Appendix $B$ 


\subsubsection{Case 1: Only heterosexual interactions.}

First consider the case where $q_{m}, q_{f}=0$, i.e. only heterosexual relations. Then (3.4) reduces to:

$$
R_{0}^{V}=\sqrt{R_{f, m}^{V} R_{m, f}^{V}}
$$

Note that $b_{f} \beta_{f, m}^{V}$ is the rate at which HSV-2 positive females infect males, while $T_{f}^{A}$ is the amount of time that a female stays in the $A_{f}$ class. Note that, in this case, the $A_{f}$ class is the only infectious class, as we assume those with latent HSV-2 are not infectious. Hence, $R_{f, m}^{V}=b_{f} \beta_{f, m}^{V} T_{f}^{A}$ is the total number of males that one HSV-2 positive female individual will infect within her sexual lifetime. Similarly, $R_{m, f}^{V}=b_{m} \beta_{m, f}^{V} T_{m}^{A}$ is the number of females that an HSV-2 positive male will infect in his sexual lifetime. Thus, 3.6 is the square root of the average number of secondary infections caused by one HSV-2 positive person through heterosexual contact.

\subsubsection{Case 2: Only homosexual interactions.}

Next, consider the case where $q_{m}, q_{f}=1$, i.e. only homosexual relations. In this case, we have eigenvalues of $F V^{-1}: R_{f, f}^{V}$ and $R_{m, m}^{V}$. Note that $R_{f, f}^{V}$ is the reproduction number for the system excluding the male population and $R_{m, m}^{V}$ is the reproduction number for the system excluding the female population. We define:

$$
R_{0}^{V}=\max \left(R_{f, f}^{V}, R_{m, m}^{V}\right)
$$

The first term represents the total number of females that one HSV-2 positive female will infect within her sexual lifetime via homosexual encounters. Similarly, the second term represents the total number of males one HSV-2 positive male individual will infect within his sexual lifetime via homosexual relationships.

\section{Invasion Reproduction Numbers}

Because the basic reproduction numbers only provide information in a population that is without the other pathogen, it is important to consider another threshold value, the invasion reproduction number. Note that the invasion reproduction number is the average number of secondary infections caused by one infected individual in a population that is completely susceptible to one pathogen, but already has the other. In this section, we find the invasion reproduction number for each disease, i.e. the reproduction number for HIV when HSV-2 is endemic, and conversely, the reproduction number for HSV-2 when HIV is endemic.

\subsection{Invasion HIV Reproduction Number $\left(R_{V}^{H}\right)$}

In this section, we will give an interpretation for the HIV invasion reproduction number in the two cases with either all heterosexual or all homosexual interaction. For a complete derivation, see Appendix C We obtain the invasion reproduction number for HIV, given by:

$$
R_{V}^{H}=\rho\left(F V^{-1}\right)
$$


For transparency, we will consider in which class individuals are infected with HIV, whether they are susceptible to both diseases or are already infected with acute or latent HSV-2, and how long individuals spend in each stage. While classifying individuals in this way leads to more terms in the expression for $R_{V}^{H}$, it provides a way to more easily interpret the expression biologically.

\subsubsection{Case 1: Only heterosexual interactions.}

First consider the case where $q_{m}, q_{f}=0$, i.e. only heterosexual relations. Then we have:

$$
R_{V}^{H}=\sqrt{R_{V, S_{f}^{*}}^{H}+R_{V, A_{f}^{*}}^{H}+R_{V, L_{f}^{*}}^{H}},
$$

where

$$
\begin{aligned}
R_{V, S_{f}^{*}}^{H}= & \frac{S_{f}^{*}}{W_{f}} b_{f} \beta_{f, m}^{H} T_{f}^{H} \frac{S_{m}^{*}}{N_{m}} b_{m} \beta_{m, f}^{H} T_{m}^{H} \frac{W_{f}}{N_{f}} \\
& +\frac{S_{f}^{*}}{W_{f}} b_{f} \beta_{f, m}^{H} T_{f}^{H} \frac{S_{m}^{*}}{N_{m}} \sigma_{m}^{H} b_{m} \beta_{m, f}^{H} p_{m}^{H \rightarrow P} T_{m}^{(P)} \frac{W_{f}}{N_{f}} \\
& +\frac{S_{f}^{*}}{W_{f}} \sigma_{f}^{H} b_{f} \beta_{f, m}^{H} p_{f}^{H \rightarrow P} T_{f}^{(P)} \frac{S_{m}^{*}}{N_{m}} b_{m} \beta_{m, f}^{H} T_{m}^{H} \frac{W_{f}}{N_{f}} \\
& +\frac{S_{f}^{*}}{W_{f}} \sigma_{f}^{H} b_{f} \beta_{f, m}^{H} p_{f}^{H \rightarrow P} T_{f}^{(P)} \frac{S_{m}^{*}}{N_{m}} \sigma_{m}^{H} b_{m} \beta_{m, f}^{H} p_{m}^{H \rightarrow P} T_{m}^{(P)} \frac{W_{f}}{N_{f}} \\
& +\frac{S_{f}^{*}}{W_{f}} b_{f} \beta_{f, m}^{H} T_{f}^{H} \frac{\delta_{m}^{A} A_{m}^{*}}{N_{m}} \sigma_{m}^{H} b_{m} \beta_{m, f}^{H} T_{m}^{(P)} \frac{W_{f}}{N_{f}} \\
& +\frac{S_{f}^{*}}{W_{f}} \sigma_{f}^{H} b_{f} \beta_{f, m}^{H} p_{f}^{H \rightarrow P} T_{f}^{(P)} \frac{\delta_{m}^{A} A_{m}^{*}}{N_{m}} \sigma_{m}^{H} b_{m} \beta_{m, f}^{H} T_{m}^{(P)} \frac{W_{f}}{N_{f}} \\
& +\frac{S_{f}^{*}}{W_{f}} b_{f} \beta_{f, m}^{H} T_{f}^{H} \frac{\delta_{m}^{L} L_{m}^{*}}{N_{m}} \sigma_{m}^{H} b_{m} \beta_{m, f}^{H} T_{m}^{(Q)} \frac{W_{f}}{N_{f}} \\
& +\frac{S_{f}^{*}}{W_{f}} \sigma_{f}^{H} b_{f} \beta_{f, m}^{H} p_{f}^{H \rightarrow P} T_{f}^{(P)} \frac{\delta_{m}^{L} L_{m}^{*}}{N_{m}} \sigma_{m}^{H} b_{m} \beta_{m, f}^{H} T_{m}^{(Q)} \frac{W_{f}}{N_{f}} \\
& +\frac{\delta_{f}^{A} A_{f}^{*}}{W_{f}} \sigma_{f}^{H} b_{f} \beta_{f, m}^{H} T_{f}^{(P)} \frac{\delta_{m}^{L} L_{m}^{*}}{N_{m}} \sigma_{m}^{H} b_{m} \beta_{m, f}^{H} T_{m}^{(Q)} \frac{W_{f}}{N_{f}} \\
R_{V, A_{f}^{*}}^{H}= & \frac{\delta_{f}^{A} A_{f}^{*}}{W_{f}} \sigma_{f}^{H} b_{f} \beta_{f, m}^{H} T_{f}^{(P)} \frac{S_{m}^{*}}{N_{m}} b_{m} \beta_{m, f}^{H} T_{m}^{H} \frac{W_{f}}{N_{f}} \\
& +\frac{\delta_{f}^{A} A_{f}^{*}}{W_{f}} \sigma_{f}^{H} b_{f} \beta_{f, m}^{H} T_{f}^{(P)} \frac{S_{m}^{*}}{N_{m}} \sigma_{m}^{H} b_{m} \beta_{m, f}^{H} p_{m}^{H \rightarrow P} T_{m}^{(P)} \frac{W_{f}}{N_{f}} \\
& +\frac{\delta_{f}^{A} A_{f}^{*}}{W_{f}} \sigma_{f}^{H} b_{f} \beta_{f, m}^{H} T_{f}^{(P)} \frac{\delta_{m}^{A} A_{m}^{*}}{N_{m}} \sigma_{m}^{H} b_{m} \beta_{m, f}^{H} T_{m}^{(P)} \frac{W_{f}}{N_{f}} \\
&
\end{aligned}
$$




$$
\begin{aligned}
R_{V, L_{f}^{*}}^{H}= & \frac{\delta_{f}^{L} L_{f}^{*}}{W_{f}} \sigma_{f}^{H} b_{f} \beta_{f, m}^{H} T_{f}^{(Q)} \frac{S_{m}^{*}}{N_{m}} b_{m} \beta_{m, f}^{H} T_{m}^{H} \frac{W_{f}}{N_{f}} \\
& +\frac{\delta_{f}^{L} L_{f}^{*}}{W_{f}} \sigma_{f}^{H} b_{f} \beta_{f, m}^{H} T_{f}^{(Q)} \frac{S_{m}^{*}}{N_{m}} \sigma_{m}^{H} b_{m} \beta_{m, f}^{H} p_{m}^{H \rightarrow P} T_{m}^{(P)} \frac{W_{f}}{N_{f}} \\
& +\frac{\delta_{f}^{L} L_{f}^{*}}{W_{f}} \sigma_{f}^{H} b_{f} \beta_{f, m}^{H} T_{f}^{(Q)} \frac{\delta_{m}^{A} A_{m}^{*}}{N_{m}} \sigma_{m}^{H} b_{m} \beta_{m, f}^{H} T_{m}^{(P)} \frac{W_{f}}{N_{f}} \\
& +\frac{\delta_{f}^{L} L_{f}^{*}}{W_{f}} \sigma_{f}^{H} b_{f} \beta_{f, m}^{H} T_{f}^{(Q)} \frac{\delta_{m}^{L} L_{m}^{*}}{N_{m}} \sigma_{m}^{H} b_{m} \beta_{m, f}^{H} T_{m}^{(Q)} \frac{W_{f}}{N_{f}}
\end{aligned}
$$

and

$$
\begin{aligned}
W_{f} & =S_{f}^{*}+\delta_{f}^{A} A_{f}^{*}+\delta_{f}^{L} L_{f}^{*}, \\
p_{i}^{H \rightarrow P} & =\frac{\delta_{i}^{H} b_{j} \beta_{j, i}^{V} \frac{A_{j}^{*}}{N_{i}}}{\delta_{i}^{H} b_{j} \beta_{j, i}^{V} \frac{A_{j}^{*}}{N_{i}}+\mu_{i}^{H}}, \\
T_{i}^{H} & =\frac{1}{\delta_{i}^{H} b_{j} \beta_{j, i}^{V} \frac{A_{j}^{*}}{N_{i}}+\mu_{i}^{H}}, \\
T_{i}^{(P)} & =\frac{\mu_{i}^{Q}+\gamma_{i}^{Q}+\omega_{i}^{P}}{\left(\mu_{i}^{P}+\omega_{i}^{P}\right)\left(\mu_{i}^{Q}+\gamma_{i}^{Q}\right)-\omega_{i}^{P} \gamma_{i}^{Q}}, \\
T_{i}^{(Q)} & =\frac{\mu_{i}^{P}+\omega_{i}^{P}+\gamma_{i}^{Q}}{\left(\mu_{i}^{P}+\omega_{i}^{P}\right)\left(\mu_{i}^{Q}+\gamma_{i}^{Q}\right)-\omega_{i}^{P} \gamma_{i}^{Q}} .
\end{aligned}
$$

First note that $W_{f}$ is the size of the population susceptible to HIV, weighted by enhancement. Also, $p_{i}^{H \rightarrow P}$ is the probability that an individual will become infected with HSV-2 while in the $H_{i}$ class, while $T_{i}^{H}$ is the length of time that an individual spends in the $H_{i}$ class. $T_{i}^{(P)}$ is the amount of time that an individual spends co-infected if he or she acquires HIV while in the acute stage of HSV-2, and is derived in Appendix C.1 Also, $T_{i}^{(Q)}$ is the amount of time that an individual spends co-infected if he or she acquires HIV while in the latent stage of HSV-2, and is derived in Appendix C.2.

Now note that the invasion reproduction number, $R_{V}^{H}$, has three main parts, corresponding to the initial HIVinfected individual coming from the completely susceptible population 4.3 or the populations that are already infected with either acute (4.4) or latent (4.5) HSV-2. It is important to consider these cases separately because an individual may acquire HIV at any stage of their HSV-2 infection.

Consider the first term of 4.3]: $\frac{S_{f}^{*}}{W_{f}} b_{f} \beta_{f, m}^{H} T_{f}^{H} \frac{S_{m}^{*}}{N_{m}} b_{m} \beta_{m, f}^{H} T_{m}^{H} \frac{W_{f}}{N_{f}}$. Note that $\frac{S_{f}^{*}}{W_{f}}=\frac{S_{f}^{*}}{S_{f}^{*}+\delta_{f}^{A} A_{f}^{*}+\delta_{f}^{L} L_{f}^{*}}$ is the weighted fraction of females who are susceptible to HIV and do not yet have HSV-2; $b_{f} \beta_{f, m}^{H}$ is the rate at which HIV-positive females infect males; and $T_{f}^{H}$ is the amount of time that a female stays in the $H_{f}$ class, without exiting due to HSV-2 infection or sexual inactivity. Thus, $\frac{S_{f}^{*}}{W_{f}} b_{f} \beta_{f, m}^{H} T_{f}^{H} \frac{S_{m}^{*}}{N_{m}}$ is the total number of $S_{m}$ males that one HIV-positive female individual will infect within her sexual lifetime, provided that she does not contract HSV2. Similarly, $b_{m} \beta_{m, f}^{H} T_{m}^{H} \frac{W_{f}}{N_{f}}$ is the number of females that an HIV-positive male will infect in his sexual lifetime if he does not acquire HSV-2. Note that $\frac{W_{f}}{N_{f}}=\frac{S_{f}^{*}}{N_{f}}+\delta_{f}^{A} \frac{A_{f}^{*}}{N_{f}}+\delta_{f}^{L} \frac{L_{f}^{*}}{N_{f}}$ indicates that the HIV-positive male may infect either a completely susceptible female (to both diseases) or a female who already has HSV-2 (either acute or latent) with enhanced susceptibility to HIV $\left(\delta_{f}^{A}, \delta_{f}^{L}\right)$. Hence, the first term of 4.3) describes an initial $S_{f}$ female 
who is infected with HIV only and infects an $S_{m}$ male; he does not contract HSV-2 and infects another female with HIV.

Note that the second term of 4.3 ,,$\frac{S_{f}^{*}}{W_{f}} b_{f} \beta_{f, m}^{H} T_{f}^{H} \frac{S_{m}^{*}}{N_{m}} \sigma_{m}^{H} b_{m} \beta_{m, f}^{H} p_{m}^{H \rightarrow P} T_{m}^{(P)} \frac{W_{f}}{N_{f}}$, has the same initial female to male infection as the first term. The remaining part differs in the term $\sigma_{m}^{H} p_{m}^{H \rightarrow P} T_{m}^{(P)}$. Note that $p_{m}^{H \rightarrow P} T_{m}^{(P)}$ is the amount of time that a male who initially has only HIV spends co-infected after contracting acute HSV-2, and $\sigma_{m}^{H}$ is the enhanced infectivity of this now co-infected male. Therefore, the second term of (4.3) describes the situation where an initial $S_{f}$ female who acquires HIV only infects an $S_{m}$ male, who then contracts HSV-2 and infects another female with HIV.

The third and fourth terms of (4.3) have similar biological meanings. The third term describes the scenario in which an initial $S_{f}$ female, who acquires HIV, contracts HSV-2, and infects an $S_{m}$ male. The male does not contract HSV-2 and infects another female with HIV. The fourth term only differs in that the male would also contract HSV-2 after his HIV infection and before infecting another female with HIV.

The fifth term of 4.3 is $\frac{S_{f}^{*}}{W_{f}} b_{f} \beta_{f, m}^{H} T_{f}^{H} \frac{\delta_{m}^{A} A_{m}^{*}}{N_{m}} \sigma_{m}^{H} b_{m} \beta_{m, f}^{H} T_{m}^{(P)} \frac{W_{f}}{N_{f}}$. Note that the female to male infection differs from the first two terms in that it has $\frac{\delta_{m}^{A} A_{m}^{*}}{N_{m}}$ instead of $\frac{S_{m}^{*}}{N_{m}}$. This indicates that the female infects a male who already has acute HSV-2 with HIV. The $\delta_{m}^{A}$ parameter is the enhanced susceptibility of this HSV-2 infected male to acquiring HIV. The remaining term $\sigma_{m}^{H} b_{m} \beta_{m, f}^{H} T_{m}^{(P)} \frac{W_{f}}{N_{f}}$ describes the co-infected male infecting a female, as $b_{m} \beta_{m, f}^{H}$ is the rate at which HIV-positive males infect females, $T_{m}^{(P)}$ is the amount of time that a male is coinfected when first experiencing the acute stage of HSV-2, $\sigma_{m}^{H}$ is the enhanced infectivity of this co-infected male, and $\frac{W_{f}}{N_{f}}$ is the weighted population of females that the male may infect. Hence the fifth term of (4.3) describes the situation where an initial female with HIV only infects an $A_{m}$ male with HIV; the male is now co-infected and infects another female with HIV.

The remaining 11 terms of (4.2) have similar interpretations and are explained in Table 4 Hence, taking the sum of these 16 possible scenarios and taking the square root to account for the interaction between females and males, we have the average number of secondary female HIV infections generated by a typical HIV-infected female in a population that is completely susceptible to HIV (in a population where the dynamics for HSV-2 are stable).

\subsubsection{Case 2: Only homosexual interactions.}

Next, consider the case where $q_{m}, q_{f}=1$, i.e. only homosexual relations. Then, if we consider the female population, we have:

$$
\begin{aligned}
R_{V}^{H}= & \frac{S_{f}^{*}}{W_{f}} b_{f} \beta_{f, f}^{H} T_{f}^{H} \frac{W_{f}}{N_{f}} \\
& +\frac{S_{f}^{*}}{W_{f}} \sigma_{f}^{H} b_{f} \beta_{f, f}^{H} p_{f}^{H \rightarrow P} T_{f}^{(P)} \frac{W_{f}}{N_{f}} \\
& +\frac{\delta_{f}^{A} A_{f}^{*}}{W_{f}} \sigma_{f}^{H} b_{f} \beta_{f, f}^{H} T_{f}^{(P)} \frac{W_{f}}{N_{f}} \\
& +\frac{\delta_{f}^{L} L_{f}^{*}}{W_{f}} \sigma_{f}^{H} b_{f} \beta_{f, f}^{H} T_{f}^{(Q)} \frac{W_{f}}{N_{f}}
\end{aligned}
$$


where $W_{f}=S_{f}^{*}+\delta_{f}^{A} A_{f}^{*}+\delta_{f}^{L} L_{f}^{*}$. Similarly, if we consider the male population, we have:

$$
\begin{aligned}
R_{V}^{H}= & \frac{S_{m}^{*}}{W_{m}} b_{m} \beta_{m, m}^{H} T_{m}^{H} \frac{W_{m}}{N_{m}} \\
& +\frac{S_{m}^{*}}{W_{m}} \sigma_{m}^{H} b_{m} \beta_{m, m}^{H} p_{m}^{H \rightarrow P} T_{m}^{(P)} \frac{W_{m}}{N_{m}} \\
& +\frac{\delta_{m}^{A} A_{m}^{*}}{W_{m}} \sigma_{m}^{H} b_{m} \beta_{m, m}^{H} T_{m}^{(P)} \frac{W_{m}}{N_{m}} \\
& +\frac{\delta_{m}^{L} L_{m}^{*}}{W_{m}} \sigma_{m}^{H} b_{m} \beta_{m, m}^{H} T_{m}^{(Q)} \frac{W_{m}}{N_{m}}
\end{aligned}
$$

For interpretation, we will consider the invasion reproduction number for the female population. In this case, $R_{V}^{H}$ has four terms. The first term of 4.7) describes the situation where an HIV-positive female, who does not acquire HSV-2, infects another female with HIV. The second term represents an HIV-positive female acquiring acute HSV-2 and then infecting another female with HIV. The third term describes a co-infected female in the acute stage of HSV-2 infecting another female with HIV. The final term indicates a co-infected female in the latent stage of HSV-2 infecting another female with HIV. The interpretation of (4.8) is analogous with males instead of females.

\subsection{Invasion $H S V-2$ Reproduction Number $\left(R_{H}^{V}\right)$}

Next, we will give an interpretation for the HSV-2 invasion reproduction number in the two cases with either all heterosexual or all homosexual interaction. For a complete derivation, see Appendix D . We obtain the invasion reproduction number for HSV-2, given by:

$$
R_{H}^{V}=\rho\left(F V^{-1}\right)
$$

\subsubsection{Case 1: Only heterosexual interactions}

First consider the case where $q_{m}, q_{f}=0$, i.e. only heterosexual relations. Then we have:

$$
R_{H}^{V}=\sqrt{R_{H, S_{f}^{*}}^{V}+R_{H, H_{f}^{*}}^{V}},
$$

where

$$
\begin{aligned}
R_{H, S_{f}^{*}}^{V}= & \frac{S_{f}^{*}}{W_{f}} b_{f} \beta_{f, m}^{V} T_{f}^{A \rightarrow A} \frac{S_{m}^{*}}{N_{m}} b_{m} \beta_{m, f}^{V} T_{m}^{A \rightarrow A} \frac{W_{f}}{N_{f}} \\
& +\frac{S_{f}^{*}}{W_{f}} b_{f} \beta_{f, m}^{V} T_{f}^{A \rightarrow A} \frac{S_{m}^{*}}{N_{m}} \sigma_{m}^{V} b_{m} \beta_{m, f}^{V} \mathbb{E}\left(A_{m}\right) p_{m}^{A \rightarrow P} T_{m}^{P \rightarrow P} \frac{W_{f}}{N_{f}} \\
& +\frac{S_{f}^{*}}{W_{f}} b_{f} \beta_{f, m}^{V} T_{f}^{A \rightarrow A} \frac{S_{m}^{*}}{N_{m}} \sigma_{m}^{V} b_{m} \beta_{m, f}^{V} p_{m}^{A \rightarrow L} \mathbb{E}\left(L_{m}\right) p_{m}^{L \rightarrow Q} T_{m}^{Q \rightarrow P} \frac{W_{f}}{N_{f}} \\
& +\frac{S_{f}^{*}}{W_{f}} b_{f} \beta_{f, m}^{V} T_{f}^{A \rightarrow A} \frac{\delta_{m}^{H} H_{m}^{*}}{N_{m}} \sigma_{m}^{V} b_{m} \beta_{m, f}^{V} T_{m}^{P \rightarrow P} \frac{W_{f}}{N_{f}} \\
& +\frac{S_{f}^{*}}{W_{f}} \sigma_{f}^{V} b_{f} \beta_{f, m}^{V} \mathbb{E}\left(A_{f}\right) p_{f}^{A \rightarrow P} T_{f}^{P \rightarrow P} \frac{S_{m}^{*}}{N_{m}} b_{m} \beta_{m, f}^{V} T_{m}^{A \rightarrow A} \frac{W_{f}}{N_{f}}
\end{aligned}
$$




$$
\begin{aligned}
+ & \frac{S_{f}^{*}}{W_{f}} \sigma_{f}^{V} b_{f} \beta_{f, m}^{V} \mathbb{E}\left(A_{f}\right) p_{f}^{A \rightarrow P} T_{f}^{P \rightarrow P} \frac{S_{m}^{*}}{N_{m}} \sigma_{m}^{V} b_{m} \beta_{m, f}^{V} \mathbb{E}\left(A_{m}\right) p_{m}^{A \rightarrow P} T_{m}^{P \rightarrow P} \frac{W_{f}}{N_{f}} \\
& +\frac{S_{f}^{*}}{W_{f}} \sigma_{f}^{V} b_{f} \beta_{f, m}^{V} \mathbb{E}\left(A_{f}\right) p_{f}^{A \rightarrow P} T_{f}^{P \rightarrow P} \frac{S_{m}^{*}}{N_{m}} \sigma_{m}^{V} b_{m} \beta_{m, f}^{V} p_{m}^{A \rightarrow L} \mathbb{E}\left(L_{m}\right) p_{m}^{L \rightarrow Q} T_{m}^{Q \rightarrow P} \frac{W_{f}}{N_{f}} \\
& +\frac{S_{f}^{*}}{W_{f}} \sigma_{f}^{V} b_{f} \beta_{f, m}^{V} \mathbb{E}\left(A_{f}\right) p_{f}^{A \rightarrow P} T_{f}^{P \rightarrow P} \frac{\delta_{m}^{H} H_{m}^{*}}{N_{m}} \sigma_{m}^{V} b_{m} \beta_{m, f}^{V} T_{m}^{P \rightarrow P} \frac{W_{f}}{N_{f}} \\
& +\frac{S_{f}^{*}}{W_{f}} \sigma_{f}^{V} b_{f} \beta_{f, m}^{V} p_{f}^{A \rightarrow L} \mathbb{E}\left(L_{f}\right) p_{f}^{L \rightarrow Q} T_{f}^{Q \rightarrow P} \frac{S_{m}^{*}}{N_{m}} b_{m} \beta_{m, f}^{V} T_{m}^{A \rightarrow A} \frac{W_{f}}{N_{f}} \\
& +\frac{S_{f}^{*}}{W_{f}} \sigma_{f}^{V} b_{f} \beta_{f, m}^{V} p_{f}^{A \rightarrow L} \mathbb{E}\left(L_{f}\right) p_{f}^{L \rightarrow Q} T_{f}^{Q \rightarrow P} \frac{S_{m}^{*}}{N_{m}} \sigma_{m}^{V} b_{m} \beta_{m, f}^{V} \mathbb{E}\left(A_{m}\right) p_{m}^{A \rightarrow P} T_{m}^{P \rightarrow P} \frac{W_{f}}{N_{f}} \\
& +\frac{S_{f}^{*}}{W_{f}} \sigma_{f}^{V} b_{f} \beta_{f, m}^{V} p_{f}^{A \rightarrow L} \mathbb{E}\left(L_{f}\right) p_{f}^{L \rightarrow Q} T_{f}^{Q \rightarrow P} \frac{S_{m}^{*}}{N_{m}} \sigma_{m}^{V} b_{m} \beta_{m, f}^{V} p_{m}^{A \rightarrow L} \mathbb{E}\left(L_{m}\right) p_{m}^{L \rightarrow Q} T_{m}^{Q \rightarrow P} \frac{W_{f}}{N_{f}} \\
& +\frac{S_{f}^{*}}{W_{f}} \sigma_{f}^{V} b_{f} \beta_{f, m}^{V} p_{f}^{A \rightarrow L} \mathbb{E}\left(L_{f}\right) p_{f}^{L \rightarrow Q} T_{f}^{Q \rightarrow P} \frac{\delta_{m}^{H} H_{m}^{*}}{N_{m}} \sigma_{m}^{V} b_{m} \beta_{m, f}^{V} T_{m}^{P \rightarrow P} \frac{W_{f}}{N_{f}}, \\
R_{H, H_{f}^{*}}^{V} & \frac{\delta_{f}^{H} H_{f}^{*}}{W_{f}} \sigma_{f}^{V} b_{f} \beta_{f, m}^{V} T_{f}^{P \rightarrow P} \frac{S_{m}^{*}}{N_{m}} b_{m} \beta_{m, f}^{V} T_{m}^{A \rightarrow A} \frac{W_{f}}{N_{f}} \\
& +\frac{\delta_{f}^{H} H_{f}^{*}}{W_{f}} \sigma_{f}^{V} b_{f} \beta_{f, m}^{V} T_{f}^{P \rightarrow P} \frac{S_{m}^{*}}{N_{m}} \sigma_{m}^{V} b_{m} \beta_{m, f}^{V} \mathbb{E}\left(A_{m}\right) p_{m}^{A \rightarrow P} T_{m}^{P \rightarrow P} \frac{W_{f}}{N_{f}} \\
& +\frac{\delta_{f}^{H} H_{f}^{*}}{W_{f}} \sigma_{f}^{V} b_{f} \beta_{f, m}^{V} T_{f}^{P \rightarrow P} \frac{S_{m}^{*}}{N_{m}} \sigma_{m}^{V} b_{m} \beta_{m, f}^{V} p_{m}^{A \rightarrow L} \mathbb{E}_{(}\left(L_{m}\right) p_{m}^{L \rightarrow Q} T_{m}^{Q \rightarrow P} \frac{W_{f}}{N_{f}} \\
& +\frac{\delta_{f}^{H} H_{f}^{*}}{W_{f}} \sigma_{f}^{V} b_{f} \beta_{f, m}^{V} T_{f}^{P \rightarrow P} \frac{\delta_{m}^{H} H_{m}^{*}}{N_{m}} \sigma_{m}^{V} b_{m} \beta_{m, f}^{V} T_{m}^{P \rightarrow P} \frac{W_{f}}{N_{f}}, \\
&
\end{aligned}
$$

and

$$
\begin{aligned}
W_{f} & =S_{f}^{*}+\delta_{f}^{H} H_{f}^{*}, \\
\mathcal{E}_{i}^{A} & =\delta_{i}^{A} q_{i} b_{i} \beta_{i, i}^{H} \frac{H_{i}^{*}}{N_{i}}+\delta_{i}^{A}\left(1-q_{j}\right) b_{j} \beta_{j, i}^{H} \frac{H_{j}^{*}}{N_{i}}+\mu_{i}^{A}+\omega_{i}^{A}, \\
\mathcal{E}_{i}^{L} & =\delta_{i}^{L} q_{i} b_{i} \beta_{i, i}^{H} \frac{H_{i}^{*}}{N_{i}}+\delta_{i}^{L}\left(1-q_{j}\right) b_{j} \beta_{j, i}^{H} \frac{H_{j}^{*}}{N_{i}}+\mu_{i}^{L}+\gamma_{i}^{L}, \\
p_{i}^{A \rightarrow L} & =\frac{\omega_{i}^{A}}{\mathcal{E}_{i}^{A}}, \\
p_{i}^{A \rightarrow P} & =\frac{\delta_{i}^{A} q_{i} b_{i} \beta_{i, i}^{H} \frac{H_{i}^{*}}{N_{i}}+\delta_{i}^{A}\left(1-q_{j}\right) b_{j} \beta_{j, i}^{H} \frac{H_{j}^{*}}{N_{i}}}{\mathcal{E}_{i}^{A}}, \\
p_{i}^{L \rightarrow Q} & =\frac{\delta_{i}^{L} q_{i} b_{i} \beta_{i, i}^{H} \frac{H_{i}^{*}}{N_{i}}+\delta_{i}^{L}\left(1-q_{j}\right) b_{j} \beta_{j, i}^{H} \frac{H_{j}^{*}}{N_{i}}}{\mathcal{E}_{i}^{L}} \\
\mathbb{E}\left(A_{i}\right) & =\mathbb{E}\left(L_{i}\right)=\frac{\mathcal{E}_{i}^{A} \mathcal{E}_{i}^{L}}{\mathcal{E}_{i}^{A} \mathcal{E}_{i}^{L}-\omega_{i}^{A} \gamma_{i}^{L}}, \\
T_{i}^{A \rightarrow A} & =\frac{\mathcal{E}_{i}^{L}}{\mathcal{E}_{i}^{A} \mathcal{E}_{i}^{L}-\omega_{i}^{A} \gamma_{i}^{L}}
\end{aligned}
$$

$T_{i}^{P \rightarrow P}$ and $T_{i}^{Q \rightarrow P}$ are as in the Appendix (see (C.2) and (C.3) and $W_{f}$ is the size of the population susceptible to HSV-2, weighted by enhancement. Note that $\mathcal{E}_{i}^{A}$ is the rate of exit from the $A_{i}$ class, while $\mathcal{E}_{i}^{L}$ is the exit rate 
from the $L_{i}$ class. Then $p_{i}^{A \rightarrow L}$ is the probability that an individual will progress to the latent stage of HSV-2 before acquiring HIV or exiting the sexually active population, $p_{i}^{A \rightarrow P}$ is the probability that an individual in the acute stage of HSV-2 will acquire HIV, and $p_{i}^{L \rightarrow Q}$ is the probability that an individual in the latent stage of HSV-2 will acquire HIV. $\mathbb{E}\left(A_{i}\right)$ is the expected number of times an individual will be in the $A_{i}$ class. Similarly, $\mathbb{E}\left(L_{i}\right)$ is the expected number of times an individual will be in the $L_{i}$ class. Finally, $T_{i}^{A \rightarrow A}$ is the overall length of time that an individual spends in the $A_{i}$ class, taking into account the progression of HSV-2 from acute to latent and back. The expressions $\mathbb{E}\left(A_{i}\right), \mathbb{E}\left(L_{i}\right)$, and $T_{i}^{A \rightarrow A}$ are derived in Appendix $\mathrm{D}$.

Note that this case is similar to the invasion reproduction number for HIV, but the interpretations differ as only individuals in the acute stage may infect others with HSV-2. Also, $R_{H}^{V}$ has two main parts corresponding to the cases in which the initial HSV-2-infected individual comes from the completely susceptible (4.11) or population that is already infected with HIV 4.12).

Consider the first term of 4.11): $\frac{S_{f}^{*}}{W_{f}} b_{f} \beta_{f, m}^{V} T_{f}^{A \rightarrow A} \frac{S_{m}^{*}}{N_{m}} b_{m} \beta_{m, f}^{V} T_{m}^{A \rightarrow A} \frac{W_{f}}{N_{f}}$. Note that $\frac{S_{f}^{*}}{W_{f}}$ is the weighted fraction of females who are susceptible to HSV-2 and do not yet have HIV; $b_{f} \beta_{f, m}^{V}$ is the rate at which HSV-2-infected females infect males; and $T_{f}^{A \rightarrow A}$ is the amount of time that a female stays in the $A_{f}$ class, without exiting due to HIV infection or sexual inactivity. Thus, $\frac{S_{f}^{*}}{W_{f}} b_{f} \beta_{f, m}^{V} T_{f}^{A \rightarrow A} \frac{S_{m}^{*}}{N_{m}}$ is the total number of $S_{m}$ males that one HSV2-infected female individual will infect within her lifetime of sexual activity, provided that she does not contract HIV. Similarly, $b_{m} \beta_{m, f}^{V} T_{m}^{A \rightarrow A} \frac{W_{f}}{N_{f}}$ is the number of females that an HSV-2-infected male will infect in his sexual lifetime if he does not acquire HIV. Note that $\frac{W_{f}}{N_{f}}=\frac{S_{f}^{*}}{N_{f}}+\delta_{f}^{H} \frac{H_{f}^{*}}{N_{f}}$ indicates that this HSV-2-infected male may infect either a completely susceptible female (to both diseases) or a female who already has HIV with an enhanced susceptibility to HSV-2 $\left(\delta_{f}^{H}\right)$. Hence, the first term of 4.11 describes an $S_{f}$ female who acquires HSV-2-only and infects an $S_{m}$ male with HSV-2; the male does not contract HIV and infects another female with HSV-2.

Now the second term of 4.11], $\frac{S_{f}^{*}}{W_{f}} b_{f} \beta_{f, m}^{V} T_{f}^{A \rightarrow A} \frac{S_{m}^{*}}{N_{m}} \sigma_{m}^{V} b_{m} \beta_{m, f}^{V} \mathbb{E}\left(A_{m}\right) p_{m}^{A \rightarrow P} T_{m}^{P \rightarrow P} \frac{W_{f}}{N_{f}}$, has the same initial female-to-male infection as the first term. The remaining part differs in the term $\sigma_{m}^{V} \mathbb{E}\left(A_{m}\right) p_{m}^{A \rightarrow P} T_{m}^{P \rightarrow P}$. Note that $\mathbb{E}\left(A_{m}\right)$ is the expected number of times that an individual will remain in the $A_{m}$ class, $p_{m}^{A \rightarrow P}$ is the probability that this $A_{m}$ individual will be infected with HIV, $T_{m}^{P \rightarrow P}$ is the amount of time that a male who initially only has HSV-2 spends co-infected and in the acute stage of HSV-2 after contracting HIV, and $\sigma_{m}^{V}$ is the enhanced infectivity of this now co-infected male. Therefore, the second term of 4.11) describes the situation where an $S_{f}$ female who acquires HSV-2-only infects an $S_{m}$ male, who then contracts HIV while in the acute stage of HSV-2 and infects another female with HSV-2.

The third term of (4.11) differs from the second again in the male infection term: $p_{m}^{A \rightarrow L} \mathbb{E}\left(L_{m}\right) p_{m}^{L \rightarrow Q} T_{m}^{Q \rightarrow P}$. This describes the scenario in which an $S_{f}$ female acquires HSV-2-only and infects an $S_{m}$ male. The male goes from the acute stage of the disease to the latent stage, acquires HIV while in the latent stage of HSV-2, and must become acute again before infecting another female with HSV-2.

The fourth term of 4.11) is $\frac{S_{f}^{*}}{W_{f}} b_{f} \beta_{f, m}^{V} T_{f}^{A \rightarrow A} \frac{\delta_{m}^{H} H_{m}^{*}}{N_{m}} \sigma_{m}^{V} b_{m} \beta_{m, f}^{V} T_{m}^{P \rightarrow P} \frac{W_{f}}{N_{f}}$. Note that the female-to-male infection differs from the first two terms in that it has $\frac{\delta_{m}^{H} H_{m}^{*}}{N_{m}}$ instead of $\frac{S_{m}^{*}}{N_{m}}$. This indicates that the female infects 
a male who already has HIV with HSV-2. The $\delta_{m}^{H}$ parameter is the enhanced susceptibility of this HIV infected male to HSV-2. The remaining term, $\sigma_{m}^{V} b_{m} \beta_{m, f}^{V} T_{m}^{P \rightarrow P} \frac{W_{f}}{N_{f}}$, describes the co-infected male infecting a female, as $b_{m} \beta_{m, f}^{V}$ is the rate at which HSV-2-infected males infect females, $T_{m}^{P \rightarrow P}$ is the amount of time that a male is co-infected and in the acute stage of HSV-2 when first experiencing the acute stage of HSV-2, $\sigma_{m}^{V}$ is the enhanced infectivity of this co-infected male, and $\frac{W_{f}}{N_{f}}$ is the weighted population of females that this male may infect. Hence the fourth term of (4.11) describes the situation where a female with HSV-2-only infects an $H_{m}$ male with HSV-2; the male is now co-infected and infects another female with HSV-2.

The remaining 12 terms of 4.10 have similar interpretations and are explained in Tables 5 and 6 Thus, taking the sum of these 16 possible scenarios and taking the square root, we have the average number of secondary female HSV-2 infections generated by a typical HSV-2-infected female in a population that is completely susceptible to HSV-2 (in a population where the dynamics for HIV are stable).

\subsubsection{Case 2: Only homosexual interactions.}

Next, consider the case where $q_{m}, q_{f}=1$, i.e. only homosexual relations. Then, if we consider the female population, we have:

$$
\begin{aligned}
R_{H}^{V}= & \frac{S_{f}^{*}}{W_{f}} b_{f} \beta_{f, f}^{V} T_{f}^{A \rightarrow A} \frac{W_{f}}{N_{f}} \\
& +\frac{S_{f}^{*}}{W_{f}} \sigma_{f}^{V} b_{f} \beta_{f, f}^{V} \mathbb{E}\left(A_{f}\right) p_{f}^{A \rightarrow P} T_{f}^{P \rightarrow P} \frac{W_{f}}{N_{f}} \\
& +\frac{S_{f}^{*}}{W_{f}} \sigma_{f}^{V} b_{f} \beta_{f, f}^{V} p_{f}^{A \rightarrow L} \mathbb{E}\left(L_{f}\right) p_{f}^{L \rightarrow Q} T_{f}^{Q \rightarrow P} \frac{W_{f}}{N_{f}} \\
& +\frac{\delta_{f}^{H} H_{f}^{*}}{W_{f}} \sigma_{f}^{V} b_{f} \beta_{f, f}^{V} T_{f}^{P \rightarrow P} \frac{W_{f}}{N_{f}},
\end{aligned}
$$

where $W_{f}=S_{f}^{*}+\delta_{f}^{H} H_{f}^{*}$. Similarly, if we consider the male population, we have:

$$
\begin{aligned}
R_{H}^{V}= & \frac{S_{m}^{*}}{W_{m}} b_{m} \beta_{m, m}^{V} T_{m}^{A \rightarrow A} \frac{W_{m}}{N_{m}} \\
& +\frac{S_{m}^{*}}{W_{m}} \sigma_{m}^{V} b_{m} \beta_{m, m}^{V} \mathbb{E}\left(A_{m}\right) p_{m}^{A \rightarrow P} T_{m}^{P \rightarrow P} \frac{W_{m}}{N_{m}} \\
& +\frac{S_{m}^{*}}{W_{m}} \sigma_{m}^{V} b_{m} \beta_{m, m}^{V} p_{m}^{A \rightarrow L} \mathbb{E}\left(L_{m}\right) p_{m}^{L \rightarrow Q} T_{m}^{Q \rightarrow P} \frac{W_{m}}{N_{m}} \\
& +\frac{\delta_{m}^{H} H_{m}^{*}}{W_{m}} \sigma_{m}^{V} b_{m} \beta_{m, m}^{V} T_{m}^{P \rightarrow P} \frac{W_{m}}{N_{m}} .
\end{aligned}
$$

For interpretation, we will consider the invasion reproduction number for the female population. In this case, $R_{H}^{V}$ has four terms. The first term of 4.14 describes the situation where an HSV-2-positive female, who does not acquire HIV, infects another female with HSV-2 (an individual must be in the acute stage of HSV-2 to infect). The second term represents an HSV-2-positive female acquiring HIV while in the acute stage of HSV-2 and then infecting another female with HSV-2. The third term indicates an HSV-2 positive female acquiring HIV while in the latent stage of HSV-2. This female now has both diseases and must become acute again before infecting another female with HSV-2. The final term describes a co-infected female infecting another female with HSV-2. 
The interpretation of 4.15 ) is analogous with males instead of females.

\section{Numerical Simulations}

This section gives numerical results of the total population dynamics for our model, as well as a sensitivity analysis of the basic and invasion reproduction numbers and prevalence of both diseases.

\subsection{Population Dynamics}

We performed numerical simulations to determine the prevalence of HIV, acute HSV-2, latent HSV-2, and (acute and latent) co-infection using our model parameters (see Table 2). We then used these values as a baseline in conducting a sensitivity analysis for the basic and invasion reproduction numbers.

Table 2: Parameter Values

\begin{tabular}{lll}
\hline Parameter & Value & References \\
\hline$N_{m}, N_{f}$ & $5 \cdot 10^{5}, 5 \cdot 10^{5}$ & \\
$\mu_{m}, \mu_{f}$ & $\frac{1}{300}, \frac{1}{300}$ & {$[9,14,17]$} \\
$\Lambda_{m}, \Lambda_{f}$ & $\mu_{m} N_{m}, \mu_{f} N_{f}$ & \\
$\delta_{m}^{A}, \delta_{f}^{A}$ & 1,1 & \\
$\delta_{m}^{L}, \delta_{f}^{L}$ & 1,1 & \\
$\delta_{m}^{H}, \delta_{f}^{H}$ & 1,1 & \\
$\sigma_{m}^{V}, \sigma_{f}^{V}$ & 1,1 & \\
$\sigma_{m}^{H}, \sigma_{f}^{H}$ & 1,1 & \\
$\omega_{m}^{A}, \omega_{f}^{A}$ & $2.678,2.083$ & {$[14,17]$} \\
$\omega_{m}^{P}, \omega_{f}^{P}$ & $1.875,1.458$ & {$[14,17]$} \\
$\gamma_{m}^{L}, \gamma_{f}^{L}$ & $0.436,0.339$ & {$[14,17]$} \\
$\gamma_{m}^{Q}, \gamma_{f}^{Q}$ & $0.469,0.365$ & {$[14,17]$} \\
$b_{m}$ & $\frac{1}{9}$ & {$[17]$} \\
$b_{f}$ & $\frac{1}{9}$ if $q_{f}=1$, otherwise $b_{f}=\frac{\left(1-q_{m}\right) b_{m} N_{m}}{\left(1-q_{f}\right) N_{f}}$ & {$[17]$} \\
$\beta_{m, m}^{H}, \beta_{m, f}^{H}$ & $0.1,0.04$ & {$[9,12,14,17]$} \\
$\beta_{f, f}^{H}, \beta_{f, m}^{H}$ & $0.02,0.025$ & {$[9,12,14,17]$} \\
$\beta_{m, m}^{V}, \beta_{m, f}^{V}$ & $0.5,0.3$ & {$[2,8,17]$} \\
$\beta_{f, f}^{V}, \beta_{f, m}^{V}$ & $0.2,0.2$ & {$[2,8,17]$} \\
$q_{m}, q_{f}$ & varies & {$[1,18,19]$} \\
\hline
\end{tabular}

First, it is important to recognize how the reproduction numbers may affect the disease dynamics (see Figures 2 and 37. In these figures, based on the chosen parameter values, we allowed the simulations to run until they stabilized at the equilibrium level. In Figure 2, we observe that, when both the basic and invasion reproduction numbers for HIV are less than one, HIV cannot survive in the population. However, when the basic reproduction number is less than one, but the invasion reproduction number is greater than one, we see that the survival of HIV depends on whether or not HSV-2 is already present in the population. In this case, when HSV-2 is present, HIV can invade the population; but when HSV-2 is absent, then HIV dies out. The same results hold for the other disease, as seen in Figure 3 

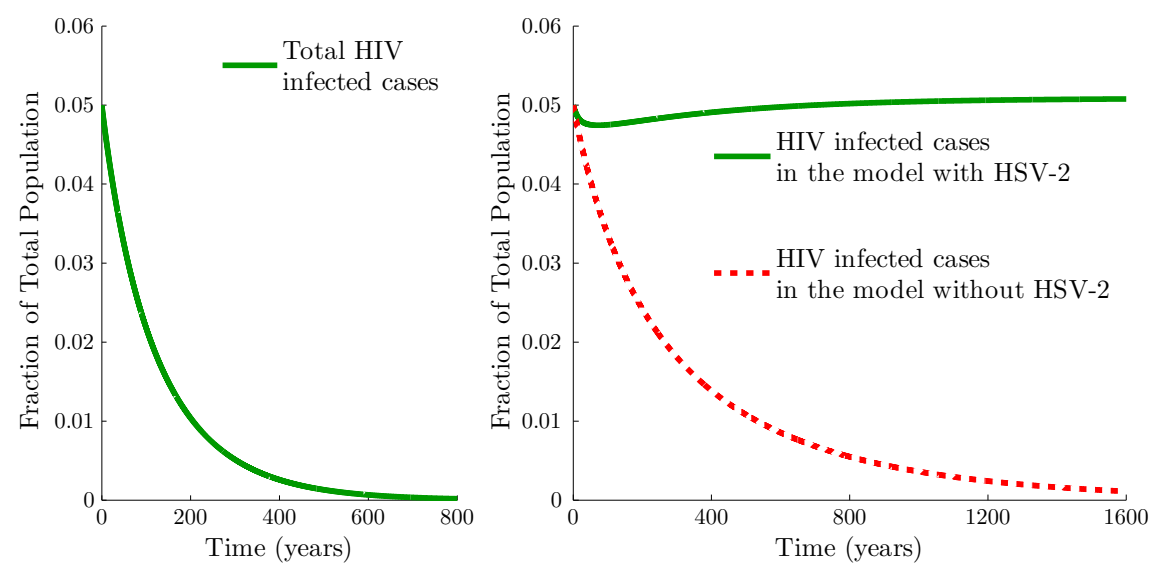

Figure 2: The HIV infected cases in the models with or without HSV-2. In the left plot, $R_{0}^{H}=0.87, R_{V}^{H}=0.97$, and $R_{0}^{V}=R_{H}^{V}=1.21$. In the right plot, $R_{0}^{H}=0.96, R_{V}^{H}=1.25$, and $R_{0}^{V}=R_{H}^{V}=1.34$.
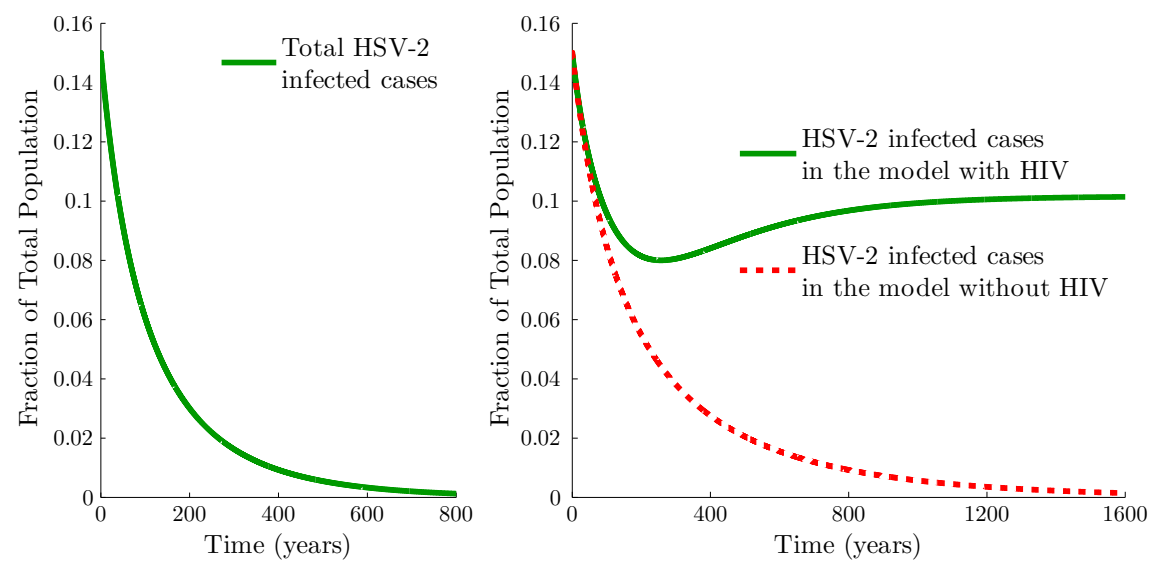

Figure 3: The HSV-2 infected cases in the models with or without HIV. In the left plot, $R_{0}^{V}=0.78, R_{H}^{V}=0.86$, and $R_{0}^{H}=R_{V}^{H}=1.14$. In the right plot, $R_{0}^{V}=0.93, R_{H}^{V}=1.12$, and $R_{0}^{H}=R_{V}^{H}=1.37$.

We will consider four main scenarios relating to the differences in sexual preference; i.e., all heterosexual individuals, all homosexual individuals, all bisexual individuals, and a final, more realistic setting, changing $q_{m}$ and $q_{f}$ accordingly. For these simulations, we will assume that the exit rates for all classes $(\mu)$ are equal, but we may relax this assumption to achieve similar results (see Section 5.3.

In Figure 4 , we see the extreme scenarios in which either the entire population is heterosexual $\left(q_{m}=q_{f}=0\right)$ or the entire population is homosexual $\left(q_{m}=q_{f}=1\right)$. These are the situations that are most often considered, but give very different results. When $q_{m}=q_{f}=0$, we see a higher prevalence of latent HSV-2 than acute HSV-2 or HIV. We may notice that, in this case, the female population has more disease overall than the male population, which is consistent with the literature [2, 20]. Furthermore, we see an overall prevalence for HSV-2 of about 15\% and an overall prevalence for HIV of around 4\%, values currently observed in the United States [1, 2]. 

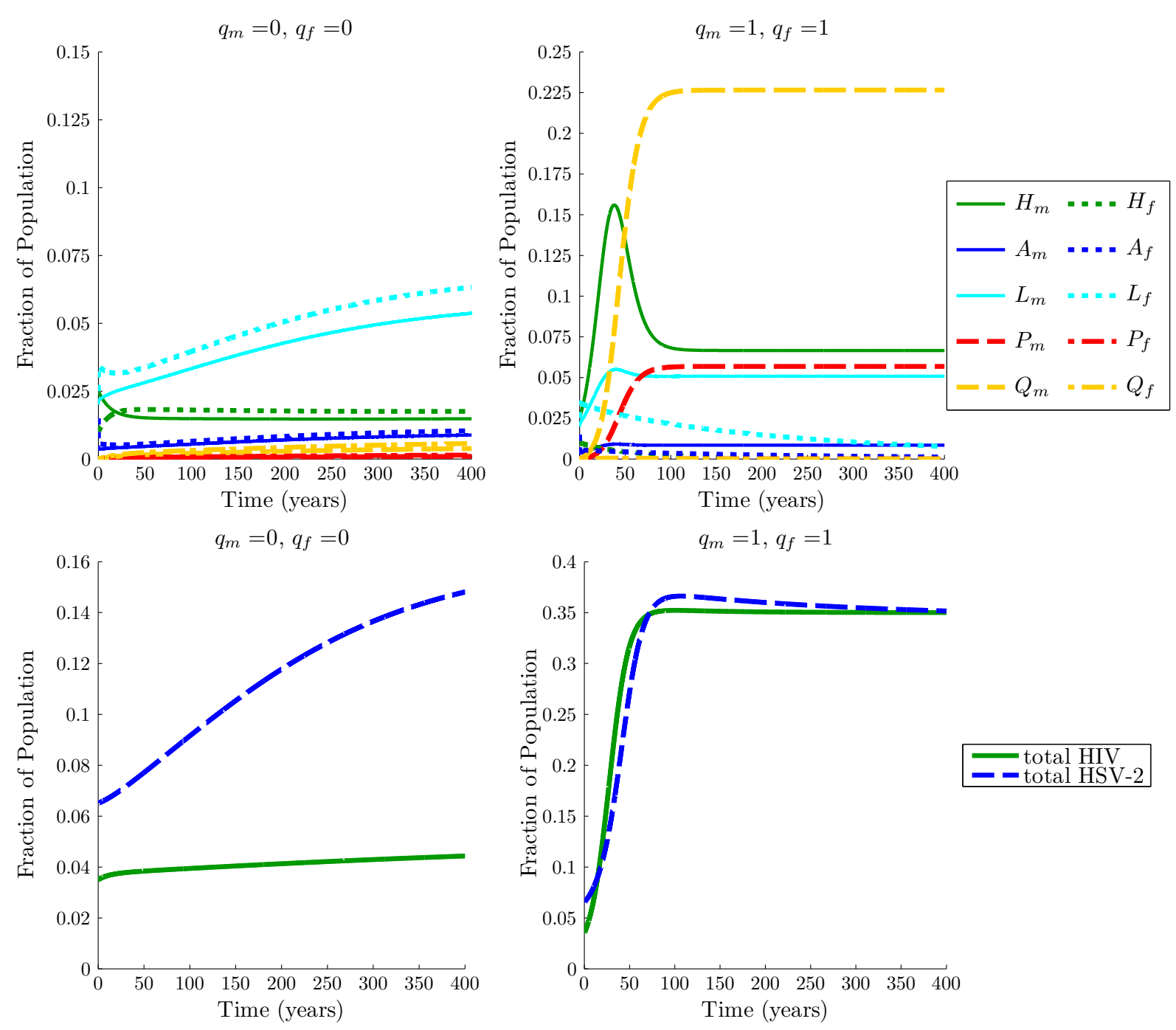

Figure 4: The fraction of the population infected with HIV-only, acute HSV-2-only, latent HSV-2-only, co-infected (acute or latent) corresponding to the two scenarios where either all individuals are heterosexual $\left(q_{m}=q_{f}=0\right)$ or all individuals are homosexual $\left(q_{m}=q_{f}=1\right)$. For $q_{m}=q_{f}=0$, we have $R_{0}^{H}=1.05, R_{0}^{V}=1.15, R_{V}^{H}=1.15$, and $R_{H}^{V}=1.20$. For $q_{m}=q_{f}=1$, we have $R_{0}^{H}=3.33, R_{0}^{V}=2.35, R_{V}^{H}=4.27$, and $R_{H}^{V}=2.35$.

When $q_{m}=q_{f}=1$, we see a much different scenario. In this case, primarily the men are infected and we see the highest prevalence among men in the co-infected latent population. This higher co-infected population causes a higher prevalence for both diseases than when all individuals are heterosexual. We also observe that, in these two extreme cases, the equilibrium is obtained in a much different time period. For $q_{m}=q_{f}=0$, the case when all individuals are heterosexual, we see that equilibrium is attained after about 300 years. But, when all individuals are homosexual $\left(q_{m}=q_{f}=1\right)$, equilibrium is reached after only about 100 years. We should take this under consideration when implementing a control policy, as there would be less time to develop a treatment strategy in the presence of a homosexual population. 

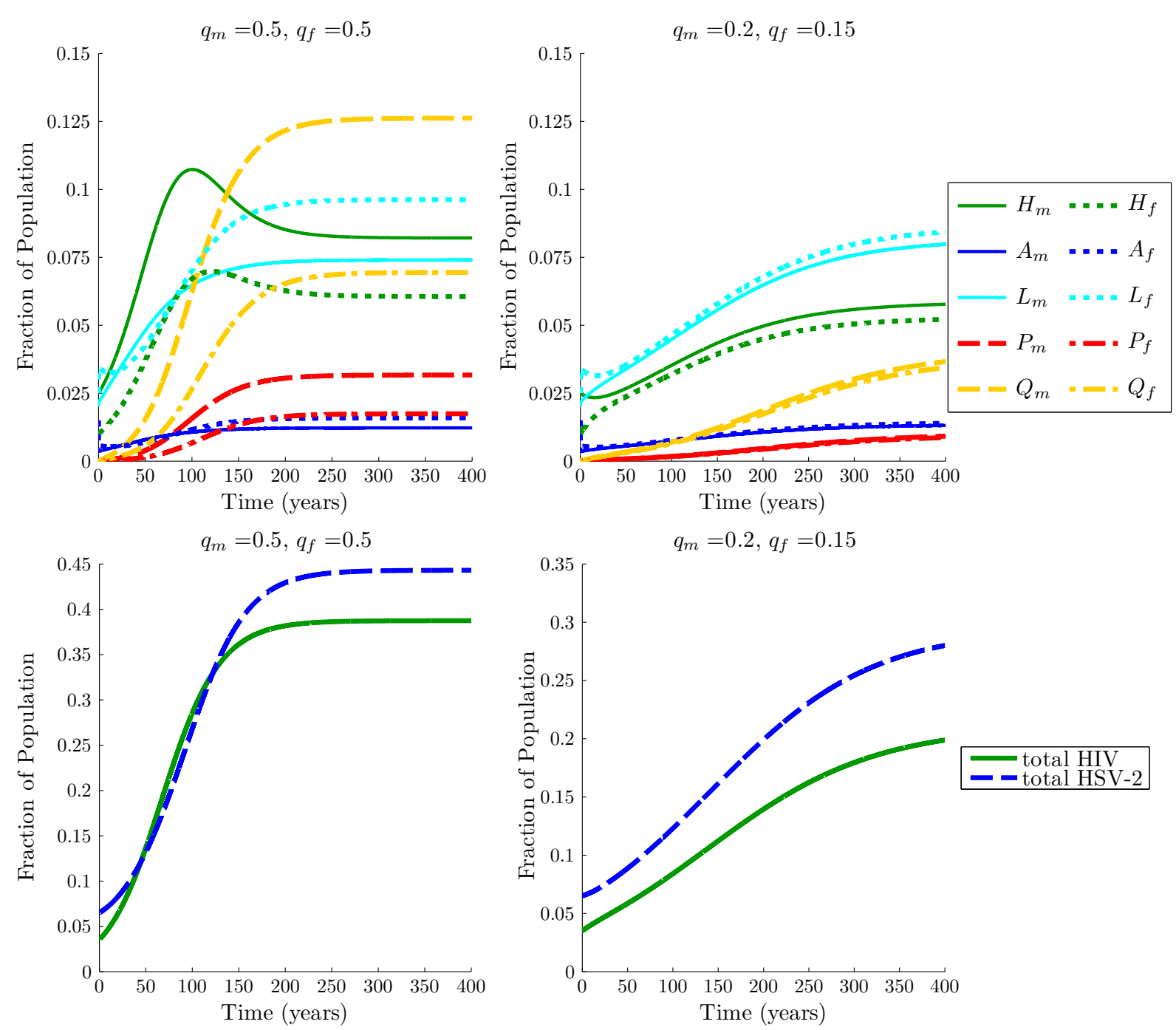

Figure 5: The fraction of the population infected with HIV-only, acute HSV-2-only, latent HSV-2-only, co-infected (acute or latent) corresponding to the two scenarios where either all individuals are bisexual $\left(q_{m}=q_{f}=0.5\right)$ or a more realistic scenario in which $q_{m}=0.2$ and $q_{f}=0.15$. For $q_{m}=q_{f}=0.5$, we have $R_{0}^{H}=1.85, R_{0}^{V}=1.50, R_{V}^{H}=2.22$, and $R_{H}^{V}=1.93$. For $q_{m}=0.2, q_{f}=0.15$, we have $R_{0}^{H}=1.27, R_{0}^{V}=1.24, R_{V}^{H}=1.44$, and $R_{H}^{V}=1.44$.

Because the extreme cases with all heterosexuals or all homosexuals give drastically different results, we want to use parameters that allow us to have both types of individuals in our model. In Figure 5, we may first look at the case where all individuals are bisexual $\left(q_{m}=q_{f}=0.5\right)$. This scenario shows an average of the previous extreme cases, but is not very realistic, as the latent co-infected population causes a significant amount of disease. Observations suggest that, in certain cities such as San Francisco, we may see levels closer to $q_{m}=0.2$ and $q_{f}=0.15\left[15\right.$, 18]. It is important to note that $q_{m}=0.2$ does not mean that $20 \%$ of the male population is homosexual. It merely means that $20 \%$ of the time, a male may choose to have relations with a member of the same sex, while this individual may or may not identify as a homosexual [21]. This case shows a higher percentage of individuals with latent co-infection and HIV-only infection than in the case where $q_{m}=q_{f}=0$, while maintaining a similar level of HSV-2-only latent infection, even though homosexual relations are infrequent. Moreover, we no 
longer see, as when $q_{m}=q_{f}=0$, that women have more disease than men. In this more realistic setting, we see that men have more HIV and hence also have more latent co-infection than women, while women continue to have higher levels of HSV-2-only infection.

In this more realistic case, there is much more latent infection among individuals with HSV-2 only rather than co-infected individuals. When comparing this prevalence to the prevalence when all individuals are heterosexual, we see a much different result. Even though the preference for homosexual contact is small, we observe an almost double HSV-2 prevalence and a much higher HIV prevalence. Hence, it is important to know where data are obtained for the prevalence of each disease and to include the interaction between and among genders to achieve a realistic result. Using a model that only incorporates heterosexual contact may significantly underestimate disease prevalences, which could lead to insufficient control efforts.

\subsection{Sensitivity Analysis}

In this section, we perform a sensitivity analysis to investigate the sensitivity of the basic and invasion reproduction numbers to our choice of model parameters. We chose a range of values and a probability density function (pdf) for each parameter and then used a method based on Latin Hypercube Sampling[22, 23] to generate the model parameters.

Table 3: Parameter Ranges

\begin{tabular}{lccc}
\hline Parameter & Lower Bound & Upper Bound & Distribution \\
\hline$q_{m}, q_{f}$ & 0 & 0.5 & $\mathrm{U}$ \\
$\mu_{m}=\mu_{f}$ & $1 / 480$ & $1 / 240$ & $\mathrm{~T}$ \\
$b_{m}$ & $1 / 12$ & $1 / 8$ & $\mathrm{U}$ \\
$N_{m}$ & $10^{5}$ & $10^{6}$ & $\mathrm{U}$ \\
$\beta_{m, m}^{H}$ & 0.05 & 0.2 & $\mathrm{~T}$ \\
$\beta_{m, f}^{H}$ & 0.03 & 0.15 & $\mathrm{~T}$ \\
$\beta_{f, m}^{H}$ & 0.02 & 0.1 & $\mathrm{~T}$ \\
$\beta_{f, f}^{H}$ & 0.005 & 0.05 & $\mathrm{~T}$ \\
$\beta_{m, m}^{V}$ & 0.3 & 0.5 & $\mathrm{~T}$ \\
$\beta_{m}^{V}, f$ & 0.1 & 0.4 & $\mathrm{~T}$ \\
$\beta_{f, m}^{V}$ & 0.1 & 0.3 & $\mathrm{~T}$ \\
$\beta_{f, f}^{V}$ & 0.05 & 0.3 & $\mathrm{~T}$ \\
$\omega_{m}^{A}$ & 2.38 & 2.98 & $\mathrm{~T}$ \\
$\omega_{f}^{A}$ & 1.78 & 2.38 & $\mathrm{~T}$ \\
$\omega_{m}^{P}$ & 1.67 & 2.08 & $\mathrm{~T}$ \\
$\omega_{f}^{P}$ & 1.25 & 1.67 & $\mathrm{~T}$ \\
$\gamma_{m}^{L}$ & 0.39 & 0.48 & $\mathrm{~T}$ \\
$\gamma_{f}^{L}$ & 0.29 & 0.39 & $\mathrm{~T}$ \\
$\gamma_{m}^{Q}$ & 0.42 & 0.52 & $\mathrm{~T}$ \\
$\gamma_{f}^{Q}$ & 0.31 & 0.42 & $\mathrm{~T}$ \\
$\delta^{H}, \delta^{A}, \delta^{L}$ & 1 & 1.1 & $\mathrm{U}$ \\
$\sigma^{H}, \sigma^{V}$ & 1 & 1.1 & $\mathrm{U}$ \\
\hline
\end{tabular}

$\mathrm{T}$ indicates a triangular distribution, with peak at the fixed value from Table 2.

$\mathrm{U}$ indicates a uniform distribution. 
In this case, we chose either a uniform $(\mathrm{U})$ or a triangular $(\mathrm{T})$ pdf for each parameter as indicated in Table 3 By sampling equally probable regions, we obtain the Latin Hypercube[8, 22, 24]. We generated 3,000 sets of parameters to consistently produce the following results.
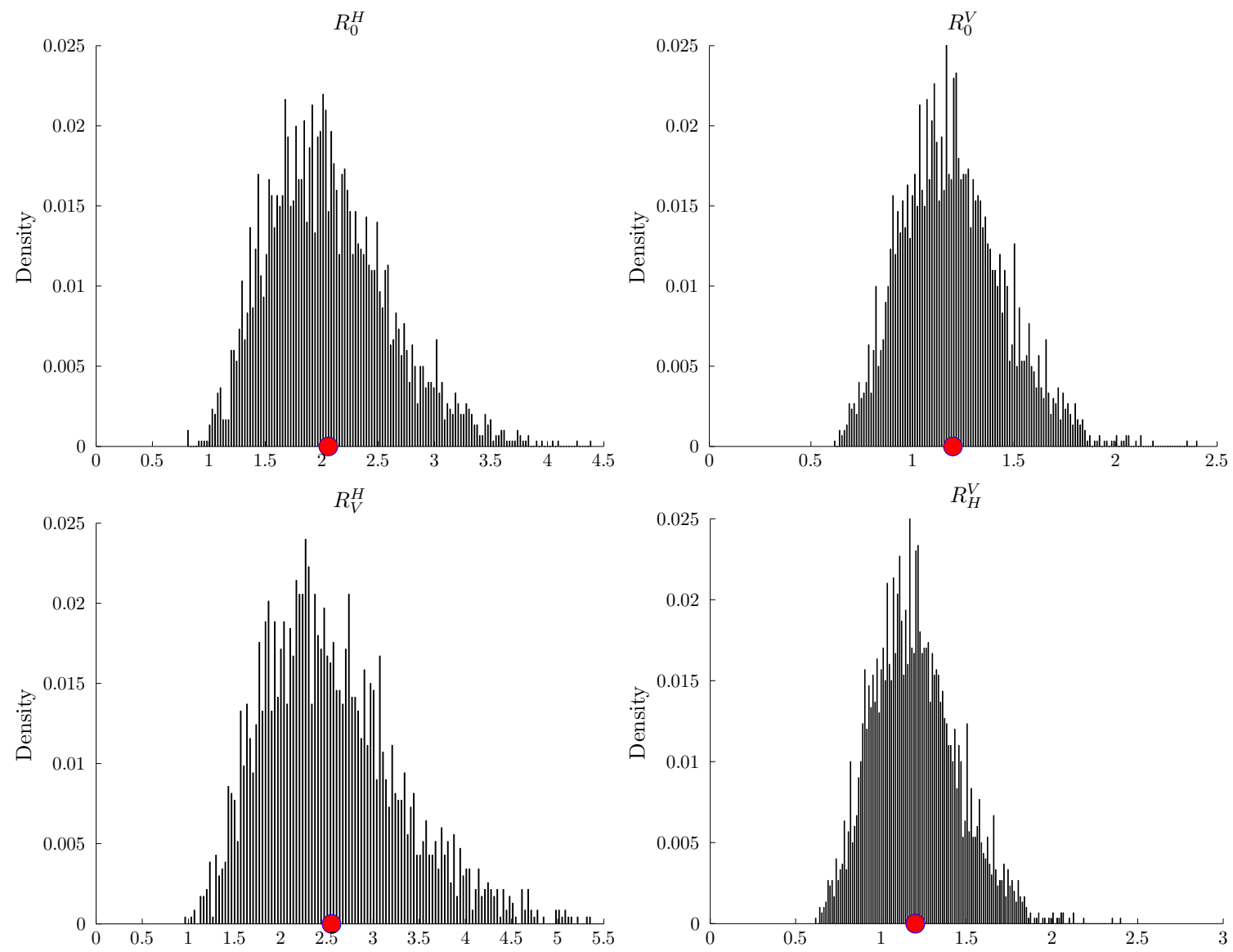

Figure 6: Densities of the basic and invasion reproduction numbers for HIV and HSV-2 obtained from Latin Hypercube parameter ranges. The point indicates the mean of the distribution.

In Figure 6, we see the densities of the basic and invasion reproduction numbers that were obtained from our parameter ranges, with their mean identified by the point on the lower axis. These reproduction number ranges are consistent with literature [8, 25, 26, 27]. In calculating the invasion reproduction numbers, we numerically calculated the equilibrium values to be used in the simulations.

Using the sets of parameter values obtained from the Latin Hypercube Sampling method, we calculated partial rank correlation coefficients (PRCC) [8, 24]. These are computed using a method that determines the effect of varying each parameter on the reproduction numbers, while accounting for the possible interactions between the remaining parameters [8, 22, 24]. The results are PRCC indices that range between -1 and 1 . A positive (negative) PRCC value indicates the relationship between the parameter and reproduction number as a positive (negative) relationship. PRCC indices may determine which parameters are most influential in their effect on the reproduction numbers, with indices of magnitude closer to 1 having the most impact[8, 22]. 
In Figures 7 and 12, we show the PRCC indices for the basic and invasion reproduction numbers. We have eliminated any parameters that have minimal effect on the reproduction numbers from the figures. First we may consider the basic reproduction numbers, $R_{0}^{H}$ and $R_{0}^{V}$ (see Figure 7). We first notice the effect of $q_{m}$ and $q_{f}$ on $R_{0}^{H}$. We see that as $q_{m}$ and $q_{f}$ increase, which increase homosexual relations in the system, we have a higher HIV basic reproduction number. We observe this result in Figures 8 and 9 . As $q_{m}$ increases in Figure 8 , the prevalence of HIV increases, primarily among the male population. Similarly, in Figure 9 we see that the levels of HIV-only increase as $q_{f}$ increases.

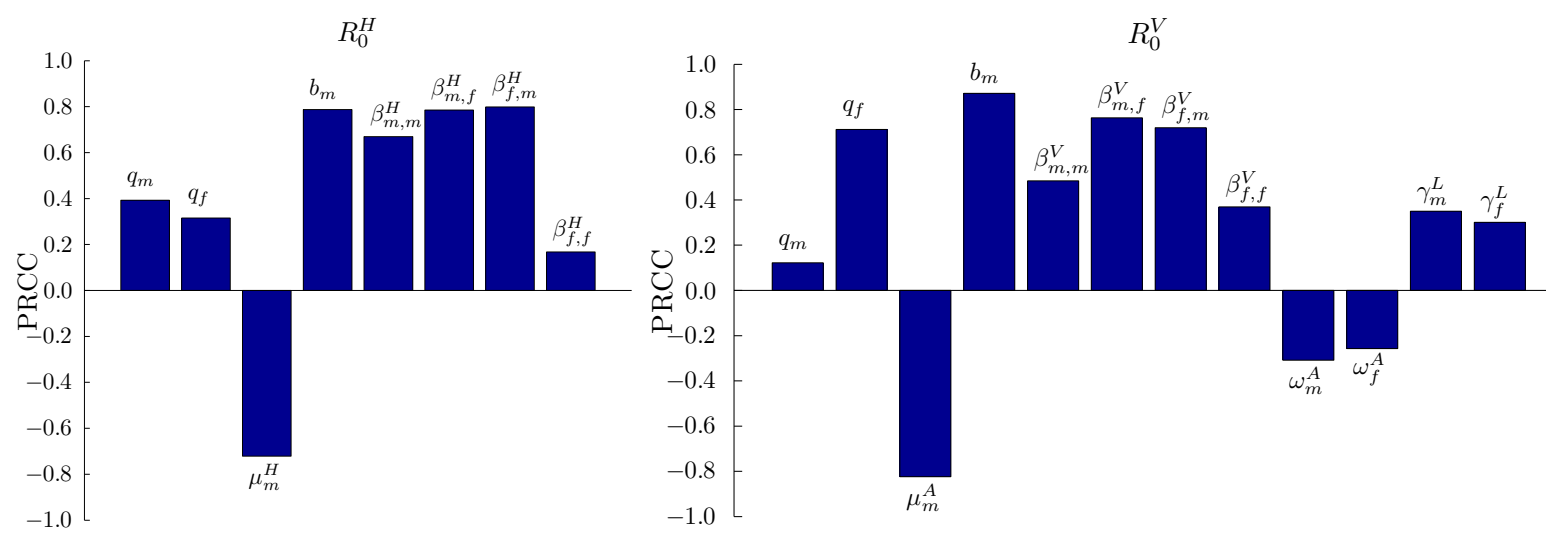

Figure 7: Partial rank correlation coefficients (PRCC) calculated using parameter ranges from Latin Hypercube Sampling with respect to the basic reproduction numbers for HIV and HSV-2. Positive (negative) values indicate a positive (negative) relationship of the parameter with the basic reproduction number. Values in magnitude closer to 1 signify parameters having the most effect on changing the reproduction number.

$R_{0}^{H}$ is more strongly influenced by the length of time spent sexually active $\left(\frac{1}{\mu^{H}}\right)$, the contact rate $b_{m}$, and the transmission probabilities for male homosexual activity and all heterosexual activity. We see that as $\mu^{H}$ increases (and hence the time spent sexually active decreases), the basic reproduction number of HIV decreases as well, which is what we would expect: with less time spent sexually active, an individual will infect fewer other individuals. The contact rate $b_{m}$ has the opposite effect, whereby an increase in an individual's number of partnerships results in a higher HIV basic reproduction number. As for the transmission rates, transmission from womanto-woman has a much lower impact than all other transmissions, with heterosexual transmission having more influence than homosexual transmission overall. This is likely due to observations which indicate that homosexual contact occurs no more than $50 \%$ of the time.

For the HSV-2 basic reproduction number (see Figure 7), we see similar results for $\mu^{V}, b_{m}$, and the transmission rates. However, we see that $q_{f}$ has a positive effect on $R_{0}^{V}$, while $q_{m}$ has a minimal effect on $R_{0}^{V}$. Comparing this result with Figures 10 and 11 , we see that this in fact holds. As $q_{m}$ increases (see Figure 10, we see relatively little change in the prevalence of HSV-2-only infection as the male population obtains more HSV-2 overall, while the female population contracts less HSV-2. For $q_{f}$ (see Figure 11, we observe that as $q_{f}$ increases, we obtain higher prevalence in both HSV-2 infected classes. This may be due to the role of $q_{f}$ in determining the contact rate $b_{f}$, as an increase in $q_{f}$ leads to an increase in $b_{f}$. 

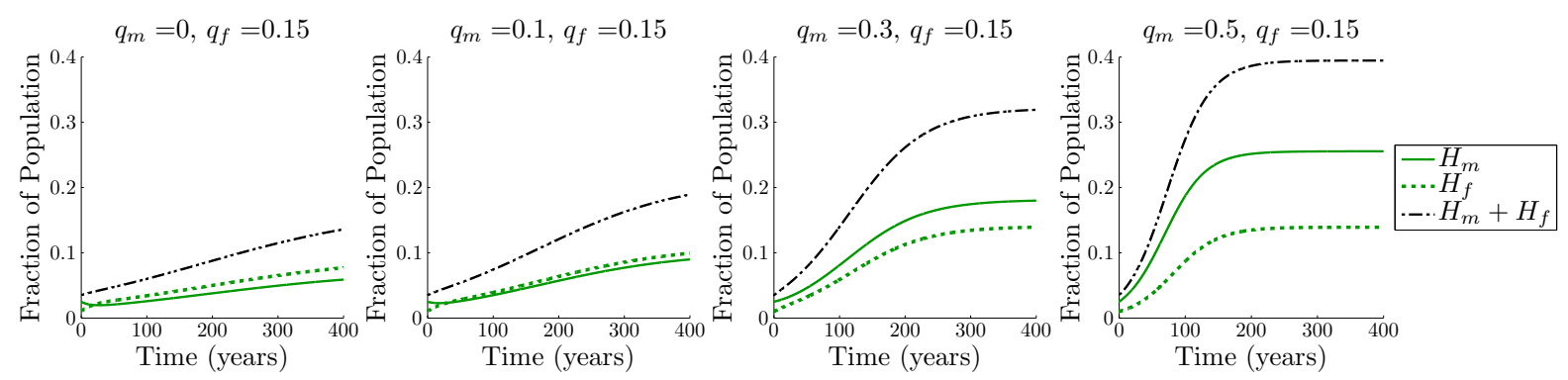

Figure 8: The fraction of the population infected with HIV-only, corresponding to different scenarios to show the effects of changing $q_{m}$ on the disease dynamics.
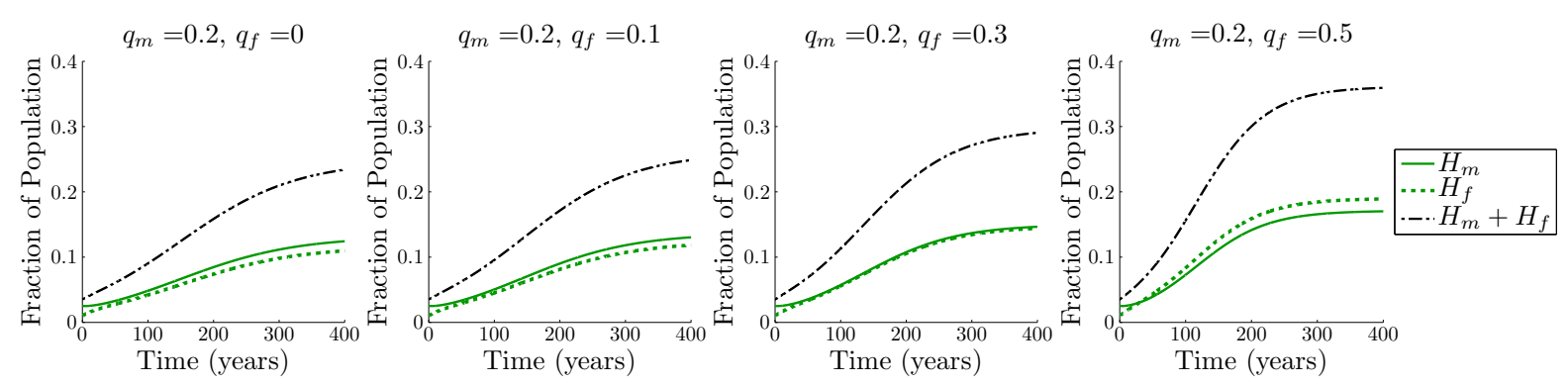

Figure 9: The fraction of the population infected with HIV-only, corresponding to different scenarios to show the effects of changing $q_{f}$ on the disease dynamics.

For the basic HSV-2 reproduction number, we also include the parameters $\omega^{A}$, which is the rate at which individuals progress to the latent stage of the disease, and $\gamma^{L}$, which is the reactivation rate. As expected, a higher rate of progression to the latent stage causes a lower $R_{0}^{V}$ as only acute individuals are infectious. Similarly, a higher reactivation rate, which introduces infectious individuals to the acute stage faster causes the basic reproduction number to increase as well.

In Figure 12, we give the PRCC indices for the invasion reproduction numbers, $R_{V}^{H}$ and $R_{H}^{V}$. The results are similar to the basic reproduction numbers, except for the added HSV-2 transmission for $R_{V}^{H}$ and the added HIV transmission for $R_{H}^{V}$. For $R_{V}^{H}$, we see that all HSV-2 transmissions have a positive effect on the invasion reproduction number. Hence, if we can implement a control measure on HSV-2, which would potentially lower the HSV-2 transmission probabilities, we would also see a reduction in $R_{V}^{H}$. However, for $R_{H}^{V}$, we see that all
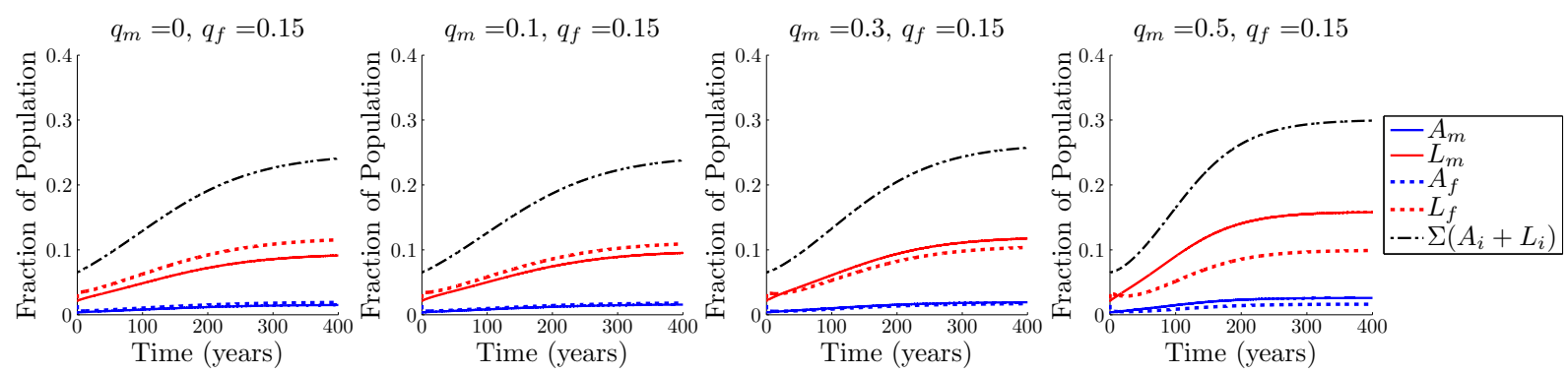

Figure 10: The fraction of the population infected with acute HSV-2 only and latent HSV-2 only corresponding to different scenarios to show the effects of changing $q_{m}$ on the disease dynamics. 

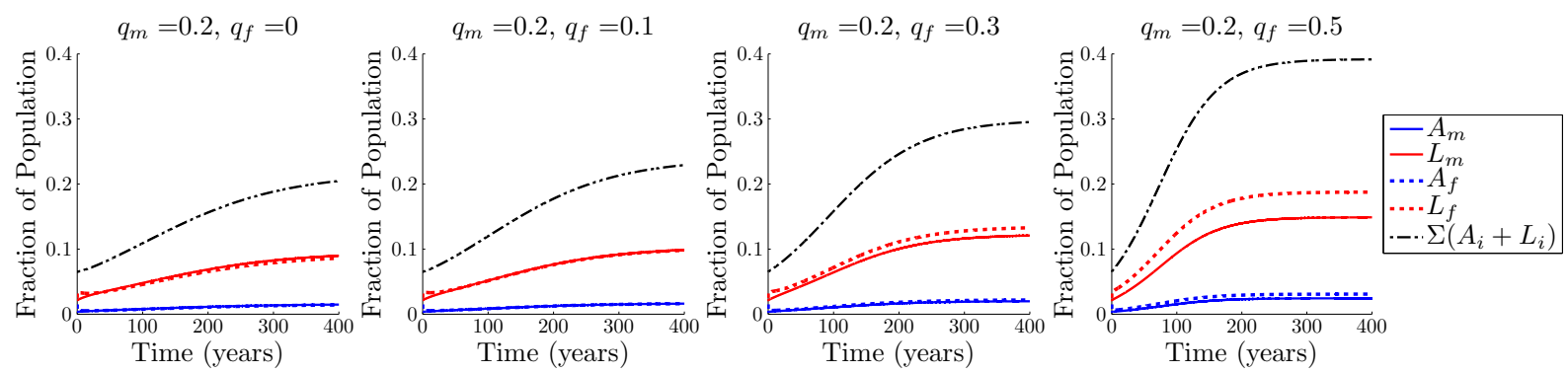

Figure 11: The fraction of the population infected with acute HSV-2 only and latent HSV-2 only corresponding to different scenarios to show the effects of changing $q_{f}$ on the disease dynamics.

HIV transmissions have very minimal effect on the possibility for HSV-2 invasion. We also notice that for $R_{H}^{V}$, the progression from acute to latent and reactivation has more impact from the singly-infected rather than co-infected population. This is likely due to the choice of parameter values, in which (as in Figure 5) the co-infected population is smaller than the HSV-2-only population.
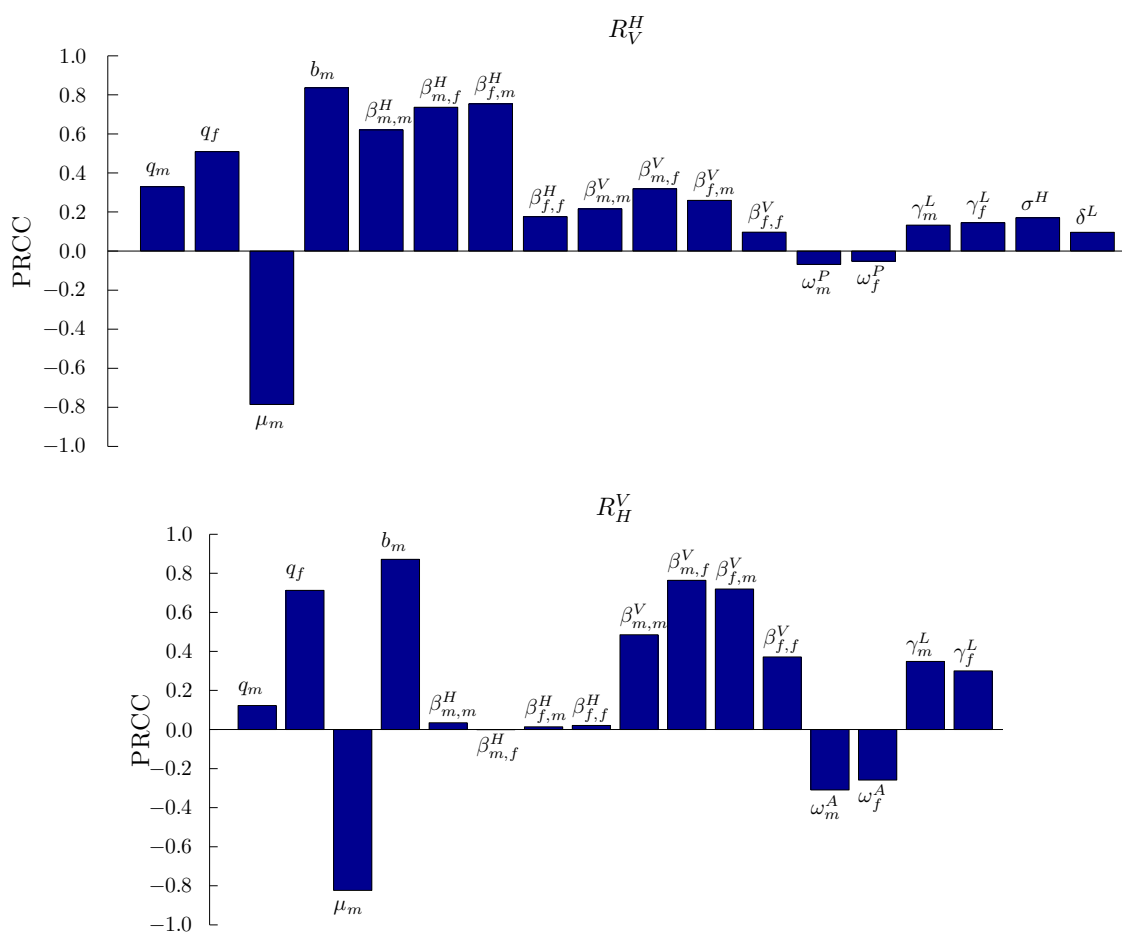

Figure 12: Partial rank correlation coefficients (PRCC) calculated using parameter ranges from Latin Hypercube Sampling with respect to the invasion reproduction numbers for HIV and HSV-2. Positive (negative) values indicate a positive (negative) relationship of the parameter with the basic reproduction number. Values in magnitude closer to 1 signify parameters having the most effect on changing the reproduction number.

Moreover, we may consider the effects of enhancement parameters. For $R_{V}^{H}$, we see that the only influential enhancement parameters are $\sigma^{H}$ and $\delta^{L}$. Recall that $\sigma^{H}$ is the enhanced infectivity of co-infected individuals when transmitting HIV and $\delta^{L}$ is the enhanced susceptibility of individuals with latent HSV-2 to HIV. The positive effect of both of these parameters on $R_{V}^{H}$ is expected as an increase in either kind of enhancement would allow 
individuals to infect or contract HIV with a higher probability, hence producing more disease in the population in a shorter period. In contrast, all of the enhancement parameters have minimal effect on $R_{H}^{V}$ and were removed from Figure 12
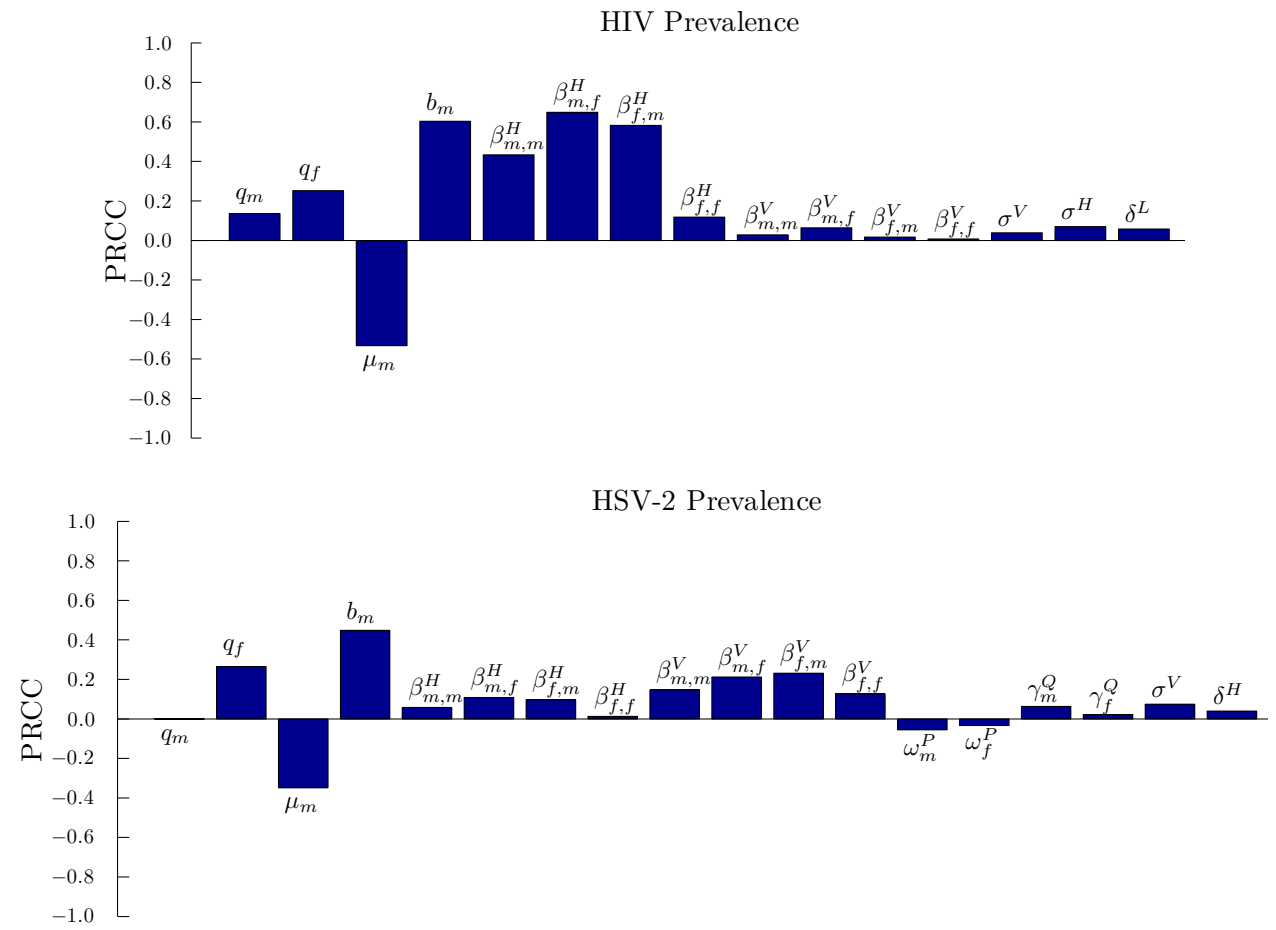

Figure 13: Partial rank correlation coefficients (PRCC) calculated using parameter ranges from Latin Hypercube Sampling with respect to the invasion reproduction numbers for HIV and HSV-2. Positive (negative) values indicate a positive (negative) relationship of the parameter with the invasion reproduction number. Values in magnitude closer to 1 signify parameters having the most effect on changing the reproduction number.

While it is important to consider the effects of the parameters on the reproduction numbers, it is also helpful to observe their effects on disease prevalence. In Figure 13 , we give the PRCC indices for both HIV and HSV-2 prevalence. As expected, many of the same parameters that affected the basic reproduction numbers for either disease also affect disease prevalence. For HSV-2 prevalence, the only influential enhancement parameters are $\sigma^{V}$ and $\delta^{H}$, which have an analogous meaning to the parameters in the PRCC for the invasion reproduction number for HIV in Figure 12. We see that all transmission parameters $(\beta)$ affect both disease prevalences positively. This indicates that treating individuals with HSV-2 will be beneficial throughout the course of both epidemics as all $\beta^{V}$ parameters have a positive impact on both invasion reproduction numbers and both disease prevalences. Hence, not only will treating individuals with HSV-2 lower the disease prevalence for both HIV and HSV-2, but it will also inhibit HIV from invading the population from the beginning, whereas treating individuals with HIV has little effect in the case of HSV-2 invasion. 


\subsection{Case with Unequal Exit Rates}

As our simulations are for the case when the exit rates from all classes are equal, we would like to consider the effect of having a higher exit rate for the HIV-infected classes. Individuals who have HIV progress to AIDS after about ten years[28], which is a much smaller amount of time than the assumed sexually active period. We conducted numerical simulations when a higher exit rate is considered for HIV-infected individuals. We observe the same underestimation in disease prevalence (primarily for HSV-2) when homosexuality is not included. We see less co-infection overall as HIV infected and co-infected individuals exit the system at a faster rate. However, the other results are all similar to the case when we assume that the exit rates are all equal. Also, the sensitivity analysis shows similar relationships of the parameters with the basic reproduction numbers and the invasion reproduction numbers as in Figures 7 and 12 The key difference in this case with different exit rates is in the sensitivity of HSV2 prevalence. When observing the role of these same parameter values on HSV-2 prevalence (see Figure 14), we observe a negative effect of the HIV transmission rates. This is likely due to the fact that decreasing $\beta^{H}$ increases the susceptible population to HSV-2, as individuals with HIV exit the system at a faster rate than completely susceptible individuals. Having more individuals susceptible to HSV-2 increases the prevalence, as we would expect.

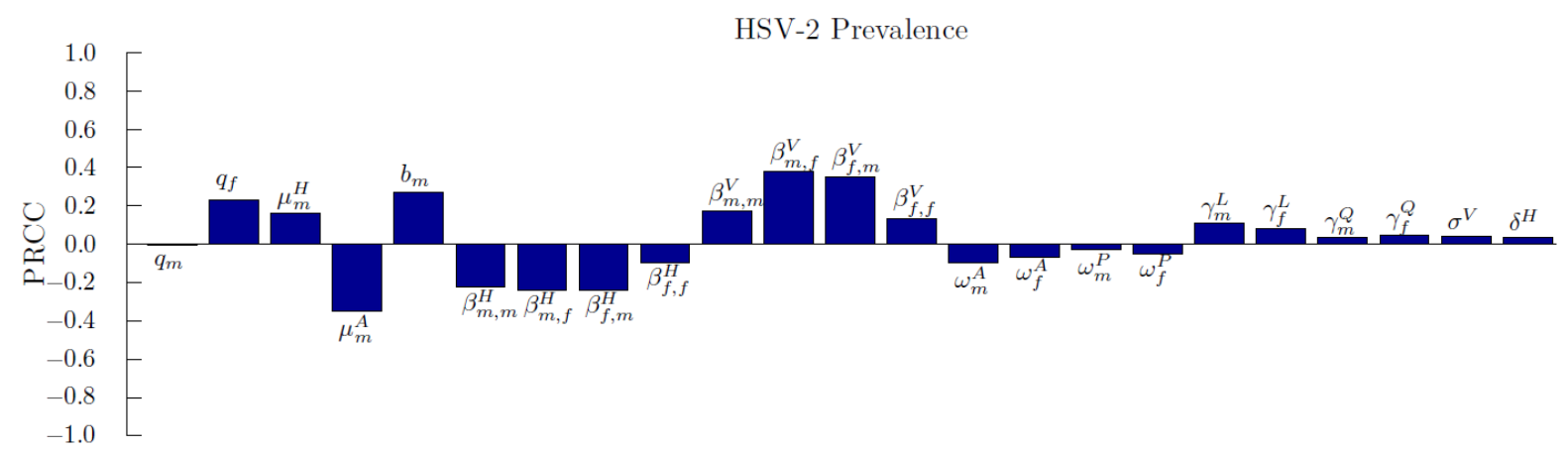

Figure 14: Partial rank correlation coefficients (PRCC) calculated using parameter ranges from Latin Hypercube Sampling with respect to the invasion reproduction number for HSV-2 when HIV-infected classes have a higher exit rate than non-HIV-infected classes. Positive (negative) values indicate a positive (negative) relationship of the parameter with the invasion reproduction number. Values in magnitude closer to 1 signify parameters having the most effect on changing the reproduction number.

\section{Discussion}

We aim to describe the coupled dynamics of HIV and HSV-2 and investigate the impact of treating patients infected with HSV-2 on the HIV epidemic. Further, we introduce a population with both heterosexual and homosexual encounters, a feature rarely considered in mathematical models. One of the main goals of our model is to investigate how the incorporation of both heterosexual and homosexual activity influences disease dynamics, either through the reproduction numbers or disease prevalences. Another goal is to determine the role of co-infection on HIV prevalence. We first calculate and interpret the basic and invasion reproduction numbers for HIV and HSV-2. 
We then perform numerical simulations to examine the population dynamics under various scenarios to determine the effect of having a mixed heterosexual and homosexual population. Finally, we analyze the reproduction numbers and disease prevalences to investigate the sensitivity of these numbers to uncertainty in the parameter values.

In looking at the population dynamics (see Figures 4 and 5, we observe a substantial difference in the models with all heterosexual and all homosexual activity. Even including a small level of homosexual activity (see Figure 5) drastically changes results from the case where all contacts are heterosexual. In this case, we see that including homosexual contacts causes the prevalence of both HIV and HSV-2 to be higher than if all individuals were heterosexual. This is primarily seen in the higher prevalence of HIV-only and latent co-infection, but all disease classes are more prevalent when $q_{m}=0.2, q_{f}=0.15$ than when $q_{m}=q_{f}=0$. This is not surprising as many of the individuals with HIV (and hence also co-infection) are members of the homosexual population[1, 6]. Thus, even though the frequency of homosexual activity may be low, ignoring this kind of interaction causes a significant underestimation of disease prevalence. When deciding on a treatment regime, this underestimation may affect which treatment strategy is optimal for any given population. Hence, it is very important to determine the frequency of homosexual activity in the population under consideration.

For the sensitivity analysis, we wish to determine which parameter values influence the reproduction numbers most (see Figures 7 and 12 . We see that a higher preference for homosexual rather than heterosexual encounters increases both basic and invasion reproduction numbers, which we confirm by observing this same relationship with respect to the disease prevalences in Figures 13 . One reason may be the effect of $q_{m}$ and $q_{f}$ in determining the contact rate $b_{f}=\frac{\left(1-q_{m}\right) b_{m} N_{m}}{\left(1-q_{f}\right) N_{f}}$. Another reason may be that the transmission rate for homosexual men is always greater than the rate among heterosexuals. In any case, it is very important to accurately estimate sexual preferences for a given population to correctly calculate the reproduction numbers.

Another key result of this model is the relationship between the transmission parameters $(\beta)$ and the reproduction numbers and disease prevalences. In Figure 12, we see that all transmission parameters increase $R_{V}^{H}$, indicating that treating either disease would inhibit HIV invasion. We may also observe the effects of these potential treatments on the disease prevalence (see Figure 13). We observe that treating either disease will be beneficial to lowering both disease prevalences. This relationship allows us to analyze various treatment strategies, whether we treat HSV-2 alone or in conjunction with HIV. Treating individuals with HSV-2 will always be helpful in controlling both HSV-2 and HIV epidemics. Particularly, treating HSV-2 cases will lower HIV and HSV-2 prevalence, while also providing an HIV-free population protection against HIV invasion.

To further explore the effects of co-infection, we may investigate the impact of enhancement on the reproduction numbers. We see that the enhanced infectivity of co-infected individuals when transmitting $\operatorname{HIV}\left(\sigma^{H}\right)$ and the enhanced susceptibility of individuals with latent HSV-2 to HIV $\left(\delta^{L}\right)$ both increase $R_{V}^{H}$ and HIV prevalence. Similarly, the enhanced infectivity of co-infected individuals when transmitting HSV-2 $\left(\sigma^{V}\right)$ and the enhanced susceptibility of individuals with HIV to HSV-2 $\left(\delta^{H}\right)$ both increase HSV-2 prevalence. This signifies that HSV-2 
facilitates the spread of HIV. Moreover, co-infection plays an important role in the spread of both diseases as the enhanced infectivity of co-infected individuals positively affects both disease prevalences (see Figure 13). The only kind of enhancement that has no impact on either disease is $\delta^{A}$, the enhanced susceptibility of individuals with acute HSV-2 to HIV. This may be due to the overall shorter time that is spent in the acute versus latent stage of HSV-2.

Due to the complexity of the current model, we modeled the choice of a heterosexual versus homosexual contact as preference (i.e. there is a probability that an interaction will be either heterosexual or homosexual in nature). Furthermore, there is little data available to indicate the appropriate sizes of the heterosexual and homosexual populations in many places, as until recently, homosexuality was illegal and/or highly looked down upon in many countries. Nevertheless, it will be important in future work to explore the effect of having a separated class for a true homosexual population, with a smaller interaction between the heterosexual and homosexual groups. Also, while implications regarding treatment strategies have been discussed, it will be interesting to compare specific treatment scenarios and their influence on the disease dynamics. This will be explored in future works.

\section{Acknowledgments}

We thank the reviewers for their helpful comments and suggestions. This research is supported in part by the NSF grant DMS-1022758.

\section{References}

[1] C. for Disease Control, Prevention, CDC fact sheet: New HIV infections in the United States.

[2] C. for Disease Control, Prevention, CDC fact sheet: Genital herpes.

[3] C. Celum, A. Wald, J. Hughes, J. Sanchez, S. Reid, S. Delany-Moretlwe, F. Cowan, M. Casapia, A. Ortiz, J. Fuchs, S. Buchbinder, B. Koblin, S. Zwerski, S. Rose, J. Wang, L. Corey, Effect of aciclovir on HIV-1 acquisition in herpes simplex virus 2 seropositive women and men who have sex with men: a randomised, double-blind, placebo-controlled trial, The Lancet 371 (9630) (2008) 2109 - 2119. doi: http://dx. doi.org/10.1016/S0140-6736(08)60920-4

URL http://www.sciencedirect.com/science/article/pii/s0140673608609204

[4] E. Freeman, H. Weiss, J. Glynn, P. Cross, J. Whitworth, R. Hayes, Herpes simplex virus 2 infection increases hiv acquisition in men and women: systematic review and meta-analysis of longitudinal studies, AIDS 20 (1) (2006) 73-83. doi:DOI:doi:10.1016/j.epidem.2010.08.003

[5] L. J. Abu-Raddad, J. T. Schiffer, R. Ashley, G. Mumtaz, R. A. Alsallaq, F. A. Akala, I. Semini, G. Riedner, D. Wilson, HSV-2 serology can be predictive of HIV epidemic potential and hidden sexual risk behavior in the middle east and north Africa, Epidemics 2 (4) (2010) 173-182. doi:DOI:doi:10.1016/j.epidem. 2010.08 .003 
[6] S. Blower, L. Ma, Calculating the contribution of herpes simplex virus type 2 epidemics to increasing HIV incidence: Treatment implications, Clinical Infectious Diseases 39 (Suppl 5) (2004) S240-S247.

[7] L. Corey, A. Wald, C. L. Celum, T. C. Quinn, The effects of herpes simplex virus-2 on HIV-1 acquisition and transmission: a review of two overlapping epidemics, JAIDS Journal of Acquired Immune Deficiency Syndromes 35 (5) (2004) 435-445.

[8] S. Blower, T. Porco, G. Darby, Predicting and preventing the emergence of antiviral drug resistance in HSV-2, Mathematical Biosciences 4.

[9] S. M. Blower, H. B. Gershengorn, R. M. Grant, A tale of two futures: HIV and antiretroviral therapy in San Francisco, Science 287.

[10] R. Granich, C. F. Gilks, C. Dye, K. M. De Cock, B. G. Williams, Universal voluntary HIV testing with immediate antiretroviral therapy as a strategy for elimination of HIV transmission: a mathematical model, The Lancet 373 (9657) (2009) 48-57.

[11] A. Foss, P. Vickerman, Z. Chalabi, P. Mayaud, M. Alary, C. Watts, Dynamic modeling of herpes simplex virus type-2 (HSV-2) transmission: Issues in structural uncertainty, Bulletin of Mathematical Biology 71 (3) (2009) 720-749. doi:10.1007/s11538-008-9379-1. URL http://dx.doi.org/10.1007/s11538-008-9379-1

[12] N. Malunguza, S. Mushayabasa, C. Chiyaka, Z. Mukandavire, Modelling the effects of condom use and antiretroviral therapy in controlling HIV/AIDS among heterosexuals, homosexuals and bisexuals, Computational and mathematical methods in medicine 11 (3) (2010) 201-222.

[13] A. Foss, P. Vickerman, P. Mayaud, H. Weiss, B. Ramesh, S. Reza-Paul, R. Washington, J. Blanchard, S. Moses, C. Lowndes, et al., Modelling the interactions between herpes simplex virus type 2 and HIV: implications for the HIV epidemic in southern India., Sexually transmitted infections 87 (1) (2011) 22-27.

[14] Z. Feng, Z. Qiu, Z. Sang, C. Lorenzo, J. Glasser, Modeling the synergy between HSV-2 and HIV and potential impact of HSV-2 therapy, Mathematical Biosciences. URL http://dx.doi.org/10.1016/j.mbs.2013.07.003

[15] G. J. Gates, Same-sex couples and the gay, lesbian, bisexual population: New estimates from the American community survey, The Williams Institute.

[16] P. van den Driessche, J. Watmough, Reproduction numbers and sub-threshold endemic equilibria for compartmental models of disease transmission, Mathematical Biosciences 180 (2002) 29-48.

[17] L. J. Abu-Raddad, A. S. Magaret, C. Celum, A. Wald, I. M. Longini, Jr., S. G. Self, L. Corey, Genital herpes has played a more important role than any other sexually transmitted infection in driving HIV prevalence in Africa, PLoS ONE 3 (5) (2008) e2230. 
[18] A. Chandra, W. D. Mosher, C. Copen, C. Sionean, Sexual behavior, sexual attraction, and sexual identity in the United States: data from the 2006-2008 national survey of family growth, National Health Statistics Reports (36).

[19] F. Xu, M. R. Sternberg, L. E. Markowitz, Women who have sex with women in the United States: Prevalence, sexual behavior and prevalence of herpes simplex virus type 2 infection: results from national health and nutrition examination survey 2001-2006, Sexually transmitted diseases 37 (7) (2010) 407-413.

[20] C. for Disease Control, Prevention, HIV surveillance report 23.

[21] F. Xu, M. R. Sternberg, L. E. Markowitz, Men who have sex with men in the United States: demographic and behavioral characteristics and prevalence of HIV and HSV-2 infection: results from national health and nutrition examination survey 2001-2006, Sexually Transmitted Diseases 37 (6) (2010) 399-405.

[22] S. Blower, H. Dowlatabadi, Sensitivity and uncertainty analysis of complex models of disease transmission: an HIV model, as an example, International Statistical Review/Revue Internationale de Statistique 62 (2) (1994) 229-243.

[23] J. Helton, J. Johnson, C. Sallaberry, C. Storlie, Survey of sampling-based methods for uncertainty and sensitivity analysis, Reliability Engineering \& System Safety 91 (10) (2006) 1175-1209.

[24] D. Hamby, A review of techniques for parameter sensitivity analysis of environmental models, Environmental Monitoring and Assessment 32 (2) (1994) 135-154.

[25] S. Blower, A. Wald, H. Gershengorn, F. Wang, L. Corey, Targeting virological core groups: A new paradigm for controlling herpes simplex virus type 2 epidemics, The Journal of Infectious Diseases 190 (2004) 16101617.

[26] T. D. Hollingsworth, R. M. Anderson, C. Fraser, HIV-1 transmission, by stage of infection., The Journal of Infectious Diseases 198 (5) (2008)687-93. doi:DOI:10.1086/590501.

URL http://www.researchgate.net/publication/23132753_HIV-1_transmission_ by_stage_of_infection

[27] J. Velasco-Hernandez, H. Gershengorn, S. Blower, Could widespread use of combination antiretroviral therapy eradicate HIV epidemics?, The Lancet infectious diseases 2 (8) (2002) 487-493.

[28] U. D. of Health \& Human Services, HIV in your body: Stages of HIV infection. 


\section{Appendix A Derivation of $R_{0}^{H}$}

We first derive the basic reproduction number for HIV. In doing so, suppose the HSV-2 and co-infected classes remain at the disease-free equilibrium, so that $A_{i}, L_{i}, P_{i}, Q_{i}=0$. In this case, system 2.11 reduces to:

$$
\left\{\begin{array}{l}
\dot{S}_{i}=\Lambda_{i}-\left(\lambda_{i, i}^{H}+\lambda_{j, i}^{H}\right) S_{i}-\mu_{i}^{S} S_{i} \\
\dot{H}_{i}=\left(\lambda_{i, i}^{H}+\lambda_{j, i}^{H}\right) S_{i}-\mu_{i}^{H} H_{i}
\end{array}\right.
$$

where the forces of infection are:

$$
\begin{aligned}
\lambda_{i, i}^{H} & =q_{i} b_{i} \beta_{i, i}^{H} \frac{H_{i}}{N_{i}} \\
\lambda_{j, i}^{H} & =\left(1-q_{j}\right) b_{j} \beta_{j, i}^{H} \frac{H_{j}}{N_{i}}, \quad i=m, f, \quad j=m, f, \quad j \neq i .
\end{aligned}
$$

We find the basic reproduction number using the approach of the next-generation matrix[16]. We construct matrix $\mathcal{F}$, which describes new infections to the system, and matrix $\mathcal{V}$, which describes all other transitions within the system.

$$
\mathcal{F}=\left(\begin{array}{c}
q_{m} b_{m} \beta_{m, m}^{H} \frac{S_{m}}{N_{m}} H_{m}+\left(1-q_{f}\right) b_{f} \beta_{f, m}^{H} \frac{S_{m}}{N_{m}} H_{f} \\
q_{f} b_{f} \beta_{f, f}^{H} \frac{S_{f}}{N_{f}} H_{f}+\left(1-q_{m}\right) b_{m} \beta_{m, f}^{H} \frac{S_{f}}{N_{f}} H_{m}
\end{array}\right) ; \quad \mathcal{V}=\left(\begin{array}{c}
\mu_{m}^{H} H_{m} \\
\mu_{f}^{H} H_{f}
\end{array}\right)
$$

Note that at the DFE, $S_{i}^{0}=N_{i}^{0}$. Then, taking partial derivatives and evaluating at the DFE, we have:

$$
F=\left(\begin{array}{cc}
q_{m} b_{m} \beta_{m, m}^{H} & \left(1-q_{f}\right) b_{f} \beta_{f, m}^{H} \\
\left(1-q_{m}\right) b_{m} \beta_{m, f}^{H} & q_{f} b_{f} \beta_{f, f}^{H}
\end{array}\right), \quad V=\left(\begin{array}{cc}
\mu_{m}^{H} & 0 \\
0 & \mu_{f}^{H}
\end{array}\right),
$$

so that

$$
F V^{-1}=\left(\begin{array}{cc}
\frac{q_{m} b_{m} \beta_{m, m}^{H}}{\mu_{m}^{H}} & \frac{\left(1-q_{f}\right) b_{f} \beta_{f, m}^{H}}{\mu_{f}^{H}} \\
\frac{\left(1-q_{m}\right) b_{m} \beta_{m, f}^{H}}{\mu_{m}^{H}} & \frac{q_{f} b_{f} \beta_{f, f}^{H}}{\mu_{f}^{H}}
\end{array}\right) .
$$

Hence the basic reproduction number (3.1) is given by:

$$
R_{0}^{H}=\rho\left(F V^{-1}\right)=\frac{1}{2}\left(R_{f, f}^{H}+R_{m, m}^{H}+\sqrt{\left(R_{f, f}^{H}-R_{m, m}^{H}\right)^{2}+4 R_{f, m}^{H} R_{m, f}^{H}}\right),
$$

where

$$
\begin{aligned}
R_{i, i}^{H} & =\frac{q_{i} b_{i} \beta_{i, i}^{H}}{\mu_{i}^{H}} \\
R_{i, j}^{H} & =\frac{\left(1-q_{i}\right) b_{i} \beta_{i, j}^{H}}{\mu_{i}^{H}}, \quad i=m, f, \quad j=m, f, \quad j \neq i .
\end{aligned}
$$




\section{Appendix B Derivation of $R_{0}^{V}$}

Next we will derive the basic reproduction number for HSV-2. Suppose that the HIV and co-infected classes remain at the disease-free equilibrium, so that $H_{i}, P_{i}, Q_{i}=0$. Then we reduce (2.1) to the system:

$$
\left\{\begin{array}{l}
\dot{S}_{i}=\Lambda_{i}-\left(\lambda_{i, i}^{V}+\lambda_{j, i}^{V}\right) S_{i}-\mu_{i}^{S} S_{i} \\
\dot{A}_{i}=\left(\lambda_{i, i}^{V}+\lambda_{j, i}^{V}\right) S_{i}+\gamma_{i}^{L} L_{i}-\left(\mu_{i}^{A}+\omega_{i}^{A}\right) A_{i} \\
\dot{L}_{i}=\omega_{i}^{A} A_{i}-\left(\mu_{i}^{L}+\gamma_{i}^{L}\right) L_{i},
\end{array}\right.
$$

where the forces of infection are:

$$
\begin{aligned}
& \lambda_{i, i}^{V}=q_{i} b_{i} \beta_{i, i}^{V} \frac{A_{i}}{N_{i}} \\
& \lambda_{j, i}^{V}=\left(1-q_{j}\right) b_{j} \beta_{j, i}^{V} \frac{A_{j}}{N_{i}}, \quad i=m, f, \quad j=m, f, \quad j \neq i .
\end{aligned}
$$

Again, using the approach of the next generation matrix, we have:

$$
\mathcal{F}=\left(\begin{array}{c}
q_{m} b_{m} \beta_{m, m}^{V} \frac{S_{m}}{N_{m}} A_{m}+\left(1-q_{f}\right) b_{f} \beta_{f, m}^{V} \frac{S_{m}}{N_{m}} A_{f} \\
q_{f} b_{f} \beta_{f, f}^{V} \frac{S_{f}}{N_{f}} A_{f}+\left(1-q_{m}\right) b_{m} \beta_{m, f}^{V} \frac{S_{f}}{N_{f}} A_{m} \\
0 \\
0
\end{array}\right) ; \quad \mathcal{V}=\left(\begin{array}{c}
\left(\mu_{m}^{A}+\omega_{m}^{A}\right) A_{m}-\gamma_{m}^{L} L_{m} \\
\left(\mu_{f}^{A}+\omega_{f}^{A}\right) A_{f}-\gamma_{f}^{L} L_{f} \\
\left(\mu_{m}^{L}+\gamma_{m}^{L}\right) L_{m}-\omega_{m}^{A} A_{m} \\
\left(\mu_{f}^{L}+\gamma_{f}^{L}\right) L_{f}-\omega_{f}^{A} A_{f}
\end{array}\right)
$$

Note that at the DFE, $S_{i}^{0}=N_{i}^{0}$. Then, taking partial derivatives and evaluating at the DFE, we have:

$$
\begin{gathered}
F=\left(\begin{array}{cccc}
q_{m} b_{m} \beta_{m, m}^{V} & \left(1-q_{f}\right) b_{f} \beta_{f, m}^{V} & 0 & 0 \\
\left(1-q_{m}\right) b_{m} \beta_{m, f}^{V} & q_{f} b_{f} \beta_{f, f}^{V} & 0 & 0 \\
0 & 0 & 0 & 0 \\
0 & 0 & 0 & 0
\end{array}\right), \\
V=\left(\begin{array}{ccccc}
\mu_{m}^{A}+\omega_{m}^{A} & 0 & -\gamma_{m}^{L} & 0 & \\
0 & \mu_{f}^{A}+\omega_{f}^{A} & 0 & -\gamma_{f}^{L} \\
-\omega_{m}^{A} & 0 & \mu_{m}^{L}+\gamma_{m}^{L} & 0 \\
0 & -\omega_{f}^{A} & 0 & \mu_{f}^{L}+\gamma_{f}^{L}
\end{array}\right),
\end{gathered}
$$

so that the basic reproduction number (3.4) is given by $\rho\left(F V^{-1}\right)$ :

$$
R_{0}^{V}=\frac{1}{2}\left(R_{f, f}^{V}+R_{m, m}^{V}+\sqrt{\left(R_{f, f}^{V}-R_{m, m}^{V}\right)^{2}+4 R_{f, m}^{V} R_{m, f}^{V}}\right),
$$

where

$$
R_{i, i}^{V}=q_{i} b_{i} \beta_{i, i}^{V} T_{i}^{A}
$$




$$
\begin{aligned}
R_{i, j}^{V} & =\left(1-q_{i}\right) b_{i} \beta_{i, j}^{V} T_{i}^{A}, \\
T_{i}^{A} & =\frac{\mu_{i}^{L}+\gamma_{i}^{L}}{\left(\mu_{i}^{A}+\omega_{i}^{A}\right)\left(\mu_{i}^{L}+\gamma_{i}^{L}\right)-\omega_{i}^{A} \gamma_{i}^{L}}, \quad i=m, f, \quad j=m, f, \quad j \neq i .
\end{aligned}
$$

Note that $T_{i}^{A}$ is the length of time that an HSV-2 infected individual spends in the acute stage of the disease, and can be derived as follows. Let $h_{1}=\frac{\omega_{i}^{A}}{\mu_{i}^{A}+\omega_{i}^{A}}$ be the probability that an HSV-2 infected individual progresses from the acute stage to the latent stage of HSV-2 before exiting the system. Similarly, let $h_{2}=\frac{\gamma_{i}^{L}}{\mu_{i}^{L}+\gamma_{i}^{L}}$ be the probability that an HSV-2 infected individual in the latent stage returns to the acute stage before exiting the system. Then the expected number of times that an HSV-2 infected individual is in the acute, rather than latent stage, is:

$$
\begin{aligned}
\mathbb{E}\left(A_{i}\right) & =\sum_{k=0}^{\infty}\left(h_{1} h_{2}\right)^{k} \\
& =\frac{1}{1-h_{1} h_{2}} \\
& =\frac{\left(\mu_{i}^{A}+\omega_{i}^{A}\right)\left(\mu_{i}^{L}+\gamma_{i}^{L}\right)}{\left(\mu_{i}^{A}+\omega_{i}^{A}\right)\left(\mu_{i}^{L}+\gamma_{i}^{L}\right)-\omega_{i}^{A} \gamma_{i}^{L}} .
\end{aligned}
$$

Note that $\mathbb{E}\left(A_{i}\right) \geq 1$ for any choice of non-negative parameters. Now, the average time spent in the $A_{i}$ class each time is $\frac{1}{\mu_{i}^{A}+\omega_{i}^{A}}$. Hence, as in 3.5 , the overall time spent in the $A_{i}$ class is:

$$
T_{i}^{A}=\frac{1}{\mu_{i}^{A}+\omega_{i}^{A}} \frac{\left(\mu_{i}^{A}+\omega_{i}^{A}\right)\left(\mu_{i}^{L}+\gamma_{i}^{L}\right)}{\left(\mu_{i}^{A}+\omega_{i}^{A}\right)\left(\mu_{i}^{L}+\gamma_{i}^{L}\right)-\omega_{i}^{A} \gamma_{i}^{L}}=\frac{\mu_{i}^{L}+\gamma_{i}^{L}}{\left(\mu_{i}^{A}+\omega_{i}^{A}\right)\left(\mu_{i}^{L}+\gamma_{i}^{L}\right)-\omega_{i}^{A} \gamma_{i}^{L}} .
$$

\section{Appendix C Derivation and Interpretation of Terms in $R_{V}^{H}$}

Here, we will derive and explain the invasion reproduction number $R_{V}^{H}$ around the HSV-2 endemic equilibrium $\left(S_{i}^{*}, 0, A_{i}^{*}, L_{i}^{*}, 0,0\right)$.

Similar to the basic reproduction numbers, we will find the invasion reproduction number $R_{V}^{H}$ using the nextgeneration approach[16]. We have:

$$
\mathcal{F}=\left(\begin{array}{c}
\left(\lambda_{m, m}^{H}+\lambda_{f, m}^{H}\right) S_{m} \\
\left(\lambda_{f, f}^{H}+\lambda_{m, f}^{H}\right) S_{f} \\
\delta_{m}^{A}\left(\lambda_{m, m}^{H}+\lambda_{f, m}^{H}\right) A_{m} \\
\delta_{f}^{A}\left(\lambda_{f, f}^{H}+\lambda_{m, f}^{H}\right) A_{f} \\
\delta_{m}^{L}\left(\lambda_{m, m}^{H}+\lambda_{f, m}^{H}\right) L_{m} \\
\delta_{f}^{L}\left(\lambda_{f, f}^{H}+\lambda_{m, f}^{H}\right) L_{f}
\end{array}\right)
$$




$$
\mathcal{V}=\left(\begin{array}{c}
\delta_{m}^{H}\left(\lambda_{m, m}^{V}+\lambda_{f, m}^{V}\right) H_{m}+\mu_{m}^{H} H_{m} \\
\delta_{f}^{H}\left(\lambda_{f, f}^{V}+\lambda_{m, f}^{V}\right) H_{f}+\mu_{f}^{H} H_{f} \\
-\delta_{m}^{H}\left(\lambda_{m, m}^{V}+\lambda_{f, m}^{V}\right) H_{m}-\gamma_{m}^{Q} Q_{m}+\left(\mu_{m}^{P}+\omega_{m}^{P}\right) P_{m} \\
-\delta_{f}^{H}\left(\lambda_{f, f}^{V}+\lambda_{m, f}^{V}\right) H_{f}-\gamma_{f}^{Q} Q_{f}+\left(\mu_{f}^{P}+\omega_{f}^{P}\right) P_{f} \\
-\omega_{m}^{P} P_{m}+\left(\mu_{m}^{Q}+\gamma_{m}^{Q}\right) Q_{m} \\
-\omega_{f}^{P} P_{f}+\left(\mu_{f}^{Q}+\gamma_{f}^{Q}\right) Q_{f}
\end{array}\right)
$$

Then, taking partial derivatives and evaluating at the endemic equilibrium, we obtain:

$$
F=\left(\begin{array}{cc}
\mathfrak{A}_{m} \frac{S_{m}^{*}}{N_{m}} & \mathfrak{B}_{m} \frac{S_{m}^{*}}{N_{m}} \\
\mathfrak{B}_{f} \frac{S_{f}^{*}}{N_{f}} & \mathfrak{A}_{f} \frac{S_{f}^{*}}{N_{f}} \\
\delta_{m}^{A} \mathfrak{A}_{m} \frac{A_{m}^{*}}{N_{m}} & \delta_{m}^{A} \mathfrak{B}_{m} \frac{A_{m}^{*}}{N_{m}} \\
\delta_{f}^{A} \mathfrak{B}_{f} \frac{A_{f}^{*}}{N_{f}} & \delta_{f}^{A} \mathfrak{A}_{f} \frac{A_{f}^{*}}{N_{f}} \\
\delta_{m}^{L} \mathfrak{A}_{m} \frac{L_{m}^{*}}{N_{m}} & \delta_{m}^{L} \mathfrak{B}_{m} \frac{L_{m}^{*}}{N_{m}} \\
\delta_{f}^{L} \mathfrak{B}_{f} \frac{L_{f}^{*}}{N_{f}} & \delta_{f}^{L} \mathfrak{A}_{f} \frac{L_{f}^{*}}{N_{f}}
\end{array}\right)\left(\begin{array}{cccccc}
1 & 0 & \sigma_{m}^{H} & 0 & \sigma_{m}^{H} & 0 \\
0 & 1 & 0 & \sigma_{f}^{H} & 0 & \sigma_{f}^{H}
\end{array}\right)
$$

and

$$
V=\left(\begin{array}{cccccc}
\mathfrak{C}_{m}+\mu_{m}^{H} & 0 & 0 & 0 & 0 & 0 \\
0 & \mathfrak{C}_{f}+\mu_{f}^{H} & 0 & 0 & 0 & 0 \\
-\mathfrak{C}_{m} & 0 & \mu_{m}^{P}+\omega_{m}^{P} & 0 & -\gamma_{m}^{Q} & 0 \\
0 & -\mathfrak{C}_{f} & 0 & \mu_{f}^{P}+\omega_{f}^{P} & 0 & -\gamma_{f}^{Q} \\
0 & 0 & -\omega_{m}^{P} & 0 & \mu_{m}^{Q}+\gamma_{m}^{Q} & 0 \\
0 & 0 & 0 & -\omega_{f}^{P} & 0 & \mu_{f}^{Q}+\gamma_{f}^{Q}
\end{array}\right)
$$

where $\mathfrak{A}_{i}=q_{i} b_{i} \beta_{i, i}^{H}, \mathfrak{B}_{i}=\left(1-q_{j}\right) b_{j} \beta_{j, i}^{H}$, and $\mathfrak{C}_{i}=\delta_{i}^{H} q_{i} b_{i} \beta_{i, i}^{V} \frac{A_{i}^{*}}{N_{i}}+\delta_{i}^{H}\left(1-q_{j}\right) b_{j} \beta_{j, i}^{V} \frac{A_{j}^{*}}{N_{i}}$, for $i=m, f, j=m, f$, $j \neq i$. Then the invasion reproduction number for HIV 4.1 is given by:

$$
R_{V}^{H}=\rho\left(F V^{-1}\right)
$$


Table 4: Biological Interpretation of Terms in $R_{V}^{H}$

\begin{tabular}{|c|c|c|}
\hline Term & Definition & Interpretation \\
\hline 1 & $\frac{S_{f}^{*}}{W_{f}} b_{f} \beta_{f, m}^{H} T_{f}^{H} \frac{S_{m}^{*}}{N_{m}} b_{m} \beta_{m, f}^{H} T_{m}^{H} \frac{W_{f}}{N_{f}}$ & $\begin{array}{l}\text { The initial } S_{f} \text { female who acquires HIV-only infects an } \\
S_{m} \text { male with HIV; the male does not contract HSV-2 and } \\
\text { infects another female with HIV. }\end{array}$ \\
\hline 2 & $\frac{S_{f}^{*}}{W_{f}} b_{f} \beta_{f, m}^{H} T_{f}^{H} \frac{S_{m}^{*}}{N_{m}} \sigma_{m}^{H} b_{m} \beta_{m, f}^{H} p_{m}^{H \rightarrow P} T_{m}^{(P)} \frac{W_{f}}{N_{f}}$ & $\begin{array}{l}\text { The initial } S_{f} \text { female who acquires HIV-only infects an } \\
S_{m} \text { male, who then contracts HSV-2 and infects another } \\
\text { female with HIV. }\end{array}$ \\
\hline 3 & $\frac{S_{f}^{*}}{W_{f}} \sigma_{f}^{H} b_{f} \beta_{f, m}^{H} p_{f}^{H \rightarrow P} T_{f}^{(P)} \frac{S_{m}^{*}}{N_{m}} b_{m} \beta_{m, f}^{H} T_{m}^{H} \frac{W_{f}}{N_{f}}$ & $\begin{array}{l}\text { The initial } S_{f} \text { female acquires HIV, contracts HSV-2, and } \\
\text { infects an } S_{m} \text { male. The male does not contract HSV-2 } \\
\text { and infects another female with HIV. }\end{array}$ \\
\hline 4 & $\frac{S_{f}^{*}}{W_{f}} \sigma_{f}^{H} b_{f} \beta_{f, m}^{H} p_{f}^{H \rightarrow P} T_{f}^{(P)} \frac{S_{m}^{*}}{N_{m}} \sigma_{m}^{H} b_{m} \beta_{m, f}^{H} p_{m}^{H \rightarrow P} T_{m}^{(P)} \frac{W_{f}}{N_{f}}$ & $\begin{array}{l}\text { The initial } S_{f} \text { female acquires HIV, contracts HSV-2, and } \\
\text { infects an } S_{m} \text { male. The male contracts HSV-2 and then } \\
\text { infects another female with HIV. }\end{array}$ \\
\hline 5 & $\frac{S_{f}^{*}}{W_{f}} b_{f} \beta_{f, m}^{H} T_{f}^{H} \frac{\delta_{m}^{A} A_{m}^{*}}{N_{m}} \sigma_{m}^{H} b_{m} \beta_{m, f}^{H} T_{m}^{(P)} \frac{W_{f}}{N_{f}}$ & $\begin{array}{l}\text { The initial female with HIV-only infects an } A_{m} \text { male with } \\
\text { HIV; he is now co-infected and infects another female } \\
\text { with HIV. }\end{array}$ \\
\hline 6 & $\frac{S_{f}^{*}}{W_{f}} \sigma_{f}^{H} b_{f} \beta_{f, m}^{H} p_{f}^{H \rightarrow P} T_{f}^{(P)} \frac{\delta_{m}^{A} A_{m}^{*}}{N_{m}} \sigma_{m}^{H} b_{m} \beta_{m, f}^{H} T_{m}^{(P)} \frac{W_{f}}{N_{f}}$ & $\begin{array}{l}\text { The initial female with HIV contracts HSV-2 and then in- } \\
\text { fects an } A_{m} \text { male with HIV. The male is now co-infected } \\
\text { and infects another female with HIV. }\end{array}$ \\
\hline 7 & $\frac{S_{f}^{*}}{W_{f}} b_{f} \beta_{f, m}^{H} T_{f}^{H} \frac{\delta_{m}^{L} L_{m}^{*}}{N_{m}} \sigma_{m}^{H} b_{m} \beta_{m, f}^{H} T_{m}^{(Q)} \frac{W_{f}}{N_{f}}$ & $\begin{array}{l}\text { A female with HIV-only infects an } L_{m} \text { male with HIV. } \\
\text { He is now co-infected and infects another female with } \\
\text { HIV. }\end{array}$ \\
\hline 8 & $\frac{S_{f}^{*}}{W_{f}} \sigma_{f}^{H} b_{f} \beta_{f, m}^{H} p_{f}^{H \rightarrow P} T_{f}^{(P)} \frac{\delta_{m}^{L} L_{m}^{*}}{N_{m}} \sigma_{m}^{H} b_{m} \beta_{m, f}^{H} T_{m}^{(Q)} \frac{W_{f}}{N_{f}}$ & $\begin{array}{l}\text { The initial female with HIV contracts HSV- } 2 \text { and then } \\
\text { infects an } L_{m} \text { male with HIV. The male now has both } \\
\text { diseases and infects another female with HIV. }\end{array}$ \\
\hline 9 & $\frac{\delta_{f}^{A} A_{f}^{*}}{W_{f}} \sigma_{f}^{H} b_{f} \beta_{f, m}^{H} T_{f}^{(P)} \frac{S_{m}^{*}}{N_{m}} b_{m} \beta_{m, f}^{H} T_{m}^{H} \frac{W_{f}}{N_{f}}$ & $\begin{array}{l}\text { A co-infected female beginning in the acute stage of } \\
\text { HSV- } 2 \text { infects an } S_{m} \text { male with HIV. The male now has } \\
\text { HIV, does not acquire HSV-2, and infects another female } \\
\text { with HIV. }\end{array}$ \\
\hline 10 & $\frac{\delta_{f}^{A} A_{f}^{*}}{W_{f}} \sigma_{f}^{H} b_{f} \beta_{f, m}^{H} T_{f}^{(P)} \frac{S_{m}^{*}}{N_{m}} \sigma_{m}^{H} b_{m} \beta_{m, f}^{H} p_{m}^{H \rightarrow P} T_{m}^{(P)} \frac{W_{f}}{N_{f}}$ & $\begin{array}{l}\text { A co-infected female in the acute HSV-2 stage infects an } \\
S_{m} \text { male with HIV. He then contracts HSV-2 and infects } \\
\text { another female with HIV. }\end{array}$ \\
\hline 11 & $\frac{\delta_{f}^{A} A_{f}^{*}}{W_{f}} \sigma_{f}^{H} b_{f} \beta_{f, m}^{H} T_{f}^{(P)} \frac{\delta_{m}^{A} A_{m}^{*}}{N_{m}} \sigma_{m}^{H} b_{m} \beta_{m, f}^{H} T_{m}^{(P)} \frac{W_{f}}{N_{f}}$ & $\begin{array}{l}\text { A co-infected female in the acute stage of HSV-2 infects } \\
\text { an } A_{m} \text { male with HIV; the male is now co-infected, and } \\
\text { he infects another female with HIV. }\end{array}$ \\
\hline 12 & $\frac{\delta_{f}^{A} A_{f}^{*}}{W_{f}} \sigma_{f}^{H} b_{f} \beta_{f, m}^{H} T_{f}^{(P)} \frac{\delta_{m}^{L} L_{m}^{*}}{N_{m}} \sigma_{m}^{H} b_{m} \beta_{m, f}^{H} T_{m}^{(Q)} \frac{W_{f}}{N_{f}}$ & $\begin{array}{l}\text { A co-infected female in the acute stage of HSV-2 infects } \\
\text { an } L_{m} \text { male with HIV. The male is now co-infected and } \\
\text { infects another female with HIV. }\end{array}$ \\
\hline 13 & $\frac{\delta_{f}^{L} L_{f}^{*}}{W_{f}} \sigma_{f}^{H} b_{f} \beta_{f, m}^{H} T_{f}^{(Q)} \frac{S_{m}^{*}}{N_{m}} b_{m} \beta_{m, f}^{H} T_{m}^{H} \frac{W_{f}}{N_{f}}$ & $\begin{array}{l}\text { A co-infected female in the latent stage of HSV-2 infects } \\
\text { an } S_{m} \text { male with HIV. The male now has HIV-only and } \\
\text { infects another female. }\end{array}$ \\
\hline 14 & $\frac{\delta_{f}^{L} L_{f}^{*}}{W_{f}} \sigma_{f}^{H} b_{f} \beta_{f, m}^{H} T_{f}^{(Q)} \frac{S_{m}^{*}}{N_{m}} \sigma_{m}^{H} b_{m} \beta_{m, f}^{H} p_{m}^{H \rightarrow P} T_{m}^{(P)} \frac{W_{f}}{N_{f}}$ & $\begin{array}{l}\text { A co-infected female in the latent stage of HSV-2 infects } \\
\text { an } S_{m} \text { male with HIV. The HIV-infected male contracts } \\
\text { HSV-2 before infecting another female with HIV. }\end{array}$ \\
\hline 15 & $\frac{\delta_{f}^{L} L_{f}^{*}}{W_{f}} \sigma_{f}^{H} b_{f} \beta_{f, m}^{H} T_{f}^{(Q)} \frac{\delta_{m}^{A} A_{m}^{*}}{N_{m}} \sigma_{m}^{H} b_{m} \beta_{m, f}^{H} T_{m}^{(P)} \frac{W_{f}}{N_{f}}$ & $\begin{array}{l}\text { A co-infected female in the latent stage of HSV-2 infects } \\
\text { a male, who has acute HSV-2, with HIV. The male now } \\
\text { has both diseases and infects another female with HIV. }\end{array}$ \\
\hline 16 & $\frac{\delta_{f}^{L} L_{f}^{*}}{W_{f}} \sigma_{f}^{H} b_{f} \beta_{f, m}^{H} T_{f}^{(Q)} \frac{\delta_{m}^{L} L_{m}^{*}}{N_{m}} \sigma_{m}^{H} b_{m} \beta_{m, f}^{H} T_{m}^{(Q)} \frac{W_{f}}{N_{f}}$ & $\begin{array}{l}\text { A co-infected female in the latent stage of HSV-2 infects } \\
\text { an } L_{m} \text { male with HIV. The male individual is now co- } \\
\text { infected and infects another female with HIV. }\end{array}$ \\
\hline
\end{tabular}




\section{C.1 Interpretation of $T_{i}^{(P)}$}

$T_{i}^{(P)}$ (see (4.6) is the amount of time that an individual spends co-infected if he or she acquires HIV while in the acute stage of HSV-2, and can be derived as follows. Let $h_{1}=\frac{\omega_{i}^{P}}{\mu_{i}^{P}+\omega_{i}^{P}}$ be the probability that a co-infected individual progresses from the acute stage to the latent stage of HSV-2 before exiting the system. Similarly, let $h_{2}=\frac{\gamma_{i}^{Q}}{\mu_{i}^{Q}+\gamma_{i}^{Q}}$ be the probability that a co-infected individual in the latent stage of HSV-2 returns to the acute stage before exiting the system. Then the expected number of times that a co-infected individual, who acquired HIV while in the acute stage of HSV-2, remains in the acute stage is:

$$
\begin{aligned}
\mathbb{E}\left(P_{i}\right) & =\sum_{k=0}^{\infty}\left(h_{1} h_{2}\right)^{k} \\
& =\frac{1}{1-h_{1} h_{2}} \\
& =\frac{\left(\mu_{i}^{P}+\omega_{i}^{P}\right)\left(\mu_{i}^{Q}+\gamma_{i}^{Q}\right)}{\left(\mu_{i}^{P}+\omega_{i}^{P}\right)\left(\mu_{i}^{Q}+\gamma_{i}^{Q}\right)-\omega_{i}^{P} \gamma_{i}^{Q}} .
\end{aligned}
$$

Note that $\mathbb{E}\left(P_{i}\right) \geq 1$ for all non-negative parameters. The average time spent in the $P_{i}$ class each time is $\frac{1}{\mu_{i}^{P}+\omega_{i}^{P}}$. Hence, the overall time spent in the $P_{i}$ class, if the individual was co-infected while in the acute stage of HSV-2, is:

$$
T_{i}^{P_{i} \rightarrow P_{i}}=\frac{1}{\mu_{i}^{P}+\omega_{i}^{P}} \frac{\left(\mu_{i}^{P}+\omega_{i}^{P}\right)\left(\mu_{i}^{Q}+\gamma_{i}^{Q}\right)}{\left(\mu_{i}^{P}+\omega_{i}^{P}\right)\left(\mu_{i}^{Q}+\gamma_{i}^{Q}\right)-\omega_{i}^{P} \gamma_{i}^{Q}}=\frac{\mu_{i}^{Q}+\gamma_{i}^{Q}}{\left(\mu_{i}^{P}+\omega_{i}^{P}\right)\left(\mu_{i}^{Q}+\gamma_{i}^{Q}\right)-\omega_{i}^{P} \gamma_{i}^{Q}} .
$$

Similarly, the expected number of times that a co-infected individual, who acquired HIV while in the acute stage of HSV-2, progresses to the latent stage is:

$$
\begin{aligned}
\mathbb{E}\left(P_{i} \rightarrow Q_{i}\right) & =h_{1} \sum_{k=0}^{\infty}\left(h_{1} h_{2}\right)^{k} \\
& =\frac{h_{1}}{1-h_{1} h_{2}} \\
& =\frac{\omega_{i}^{P}\left(\mu_{i}^{Q}+\gamma_{i}^{Q}\right)}{\left(\mu_{i}^{P}+\omega_{i}^{P}\right)\left(\mu_{i}^{Q}+\gamma_{i}^{Q}\right)-\omega_{i}^{P} \gamma_{i}^{Q}},
\end{aligned}
$$

where $\mathbb{E}\left(P_{i} \rightarrow Q_{i}\right) \geq 0$ for all non-negative parameters. The average time spent in the $Q_{i}$ class each time is $\frac{1}{\mu_{i}^{Q}+\gamma_{i}^{Q}}$. Thus, the overall time spent in the $Q_{i}$ class, if the individual was co-infected while in the acute stage of $\mathrm{HSV}-2$, is:

$$
T_{i}^{P_{i} \rightarrow Q_{i}}=\frac{1}{\mu_{i}^{Q}+\gamma_{i}^{Q}} \frac{\omega_{i}^{P}\left(\mu_{i}^{Q}+\gamma_{i}^{Q}\right)}{\left(\mu_{i}^{P}+\omega_{i}^{P}\right)\left(\mu_{i}^{Q}+\gamma_{i}^{Q}\right)-\omega_{i}^{P} \gamma_{i}^{Q}}=\frac{\omega_{i}^{P}}{\left(\mu_{i}^{P}+\omega_{i}^{P}\right)\left(\mu_{i}^{Q}+\gamma_{i}^{Q}\right)-\omega_{i}^{P} \gamma_{i}^{Q}} .
$$

Hence, as in 4.6, the length of time that an individual spends co-infected, if he or she acquires HIV while in the acute stage of HSV-2, is:

$$
T_{i}^{(P)}=T_{i}^{P_{i} \rightarrow P_{i}}+T_{i}^{P_{i} \rightarrow Q_{i}}=\frac{\mu_{i}^{Q}+\gamma_{i}^{Q}+\omega_{i}^{P}}{\left(\mu_{i}^{P}+\omega_{i}^{P}\right)\left(\mu_{i}^{Q}+\gamma_{i}^{Q}\right)-\omega_{i}^{P} \gamma_{i}^{Q}} .
$$




\section{C.2 Interpretation of $T_{i}^{(Q)}$}

In a similar way, we can see that $T_{i}^{(Q)}$ (see (4.6) is the amount of time that an individual spends co-infected if he or she acquires HIV while in the latent stage of HSV-2, and can be derived as follows. With the same $h_{1}$ and $h_{2}$ as before, the expected number of times that a co-infected individual, who acquired HIV while in the latent stage of HSV-2, remains in the latent stage is:

$$
\begin{aligned}
\mathbb{E}\left(Q_{i}\right) & =\sum_{k=0}^{\infty}\left(h_{1} h_{2}\right)^{k} \\
& =\frac{1}{1-h_{1} h_{2}} \\
& =\frac{\left(\mu_{i}^{P}+\omega_{i}^{P}\right)\left(\mu_{i}^{Q}+\gamma_{i}^{Q}\right)}{\left(\mu_{i}^{P}+\omega_{i}^{P}\right)\left(\mu_{i}^{Q}+\gamma_{i}^{Q}\right)-\omega_{i}^{P} \gamma_{i}^{Q}} .
\end{aligned}
$$

Again, $\mathbb{E}\left(Q_{i}\right) \geq 1$ for any non-negative parameter values. The average time spent in the $Q_{i}$ class each time is $\frac{1}{\mu_{i}^{Q}+\gamma_{i}^{Q}}$. Hence, the overall time spent in the $Q_{i}$ class, if he or she was co-infected while in the latent stage of $\mathrm{HSV}-2$, is:

$$
T_{i}^{Q_{i} \rightarrow Q_{i}}=\frac{1}{\mu_{i}^{Q}+\gamma_{i}^{Q}} \frac{\left(\mu_{i}^{P}+\omega_{i}^{P}\right)\left(\mu_{i}^{Q}+\gamma_{i}^{Q}\right)}{\left(\mu_{i}^{P}+\omega_{i}^{P}\right)\left(\mu_{i}^{Q}+\gamma_{i}^{Q}\right)-\omega_{i}^{P} \gamma_{i}^{Q}}=\frac{\mu_{i}^{P}+\omega_{i}^{P}}{\left(\mu_{i}^{P}+\omega_{i}^{P}\right)\left(\mu_{i}^{Q}+\gamma_{i}^{Q}\right)-\omega_{i}^{P} \gamma_{i}^{Q}} .
$$

Similarly, the expected number of times that a co-infected individual, who acquired HIV while in the latent stage of HSV-2, progresses to the acute stage is:

$$
\begin{aligned}
\mathbb{E}\left(Q_{i} \rightarrow P_{i}\right) & =h_{2} \sum_{k=0}^{\infty}\left(h_{1} h_{2}\right)^{k} \\
& =\frac{h_{2}}{1-h_{1} h_{2}} \\
& =\frac{\gamma_{i}^{Q}\left(\mu_{i}^{P}+\omega_{i}^{P}\right)}{\left(\mu_{i}^{P}+\omega_{i}^{P}\right)\left(\mu_{i}^{Q}+\gamma_{i}^{Q}\right)-\omega_{i}^{P} \gamma_{i}^{Q}},
\end{aligned}
$$

where $\mathbb{E}\left(Q_{i} \rightarrow P_{i}\right) \geq 0$. The average time spent in the $P_{i}$ class each time is $\frac{1}{\mu_{i}^{P}+\omega_{i}^{P}}$. Thus, the overall time spent in the $P_{i}$ class, if he or she was co-infected while in the latent stage of HSV-2, is:

$$
T_{i}^{Q_{i} \rightarrow P_{i}}=\frac{1}{\mu_{i}^{P}+\omega_{i}^{P}} \frac{\gamma_{i}^{Q}\left(\mu_{i}^{P}+\omega_{i}^{P}\right)}{\left(\mu_{i}^{P}+\omega_{i}^{P}\right)\left(\mu_{i}^{Q}+\gamma_{i}^{Q}\right)-\omega_{i}^{P} \gamma_{i}^{Q}}=\frac{\gamma_{i}^{Q}}{\left(\mu_{i}^{P}+\omega_{i}^{P}\right)\left(\mu_{i}^{Q}+\gamma_{i}^{Q}\right)-\omega_{i}^{P} \gamma_{i}^{Q}} .
$$

Hence, as in 4.6, the length of time that an individual spends co-infected, if he or she acquires HIV while in the latent stage of HSV-2, is:

$$
T_{i}^{(Q)}=T_{i}^{Q_{i} \rightarrow Q_{i}}+T_{i}^{Q_{i} \rightarrow P_{i}}=\frac{\mu_{i}^{P}+\omega_{i}^{P}+\gamma_{i}^{Q}}{\left(\mu_{i}^{P}+\omega_{i}^{P}\right)\left(\mu_{i}^{Q}+\gamma_{i}^{Q}\right)-\omega_{i}^{P} \gamma_{i}^{Q}} .
$$




\section{Appendix D Derivation and Interpretation of Terms in $R_{H}^{V}$}

Next, we will derive the invasion reproduction number $R_{H}^{V}$ around the HIV endemic equilibrium $\left(S_{i}^{*}, H_{i}^{*}, 0,0,0,0\right)$. Using the next-generation matrix approach, we have:

$$
\begin{aligned}
& \mathcal{F}=\left(\begin{array}{c}
\left(\lambda_{m, m}^{V}+\lambda_{f, m}^{V}\right) S_{m} \\
\left(\lambda_{f, f}^{V}+\lambda_{m, f}^{V}\right) S_{f} \\
0 \\
0 \\
\delta_{m}^{H}\left(\lambda_{m, m}^{V}+\lambda_{f, m}^{V}\right) H_{m} \\
\delta_{f}^{H}\left(\lambda_{f, f}^{V}+\lambda_{m, f}^{V}\right) H_{f} \\
0 \\
0
\end{array}\right) \\
& \mathcal{V}=\left(\begin{array}{c}
\delta_{m}^{A}\left(\lambda_{m, m}^{H}+\lambda_{f, m}^{H}\right) A_{m}-\gamma_{m}^{L} L_{m}+\left(\mu_{m}^{A}+\omega_{m}^{A}\right) A_{m} \\
\delta_{f}^{A}\left(\lambda_{f, f}^{H}+\lambda_{m, f}^{H}\right) A_{f}-\gamma_{f}^{L} L_{f}+\left(\mu_{f}^{A}+\omega_{m}^{A}\right) A_{f} \\
-\omega_{m}^{A} A_{m}+\delta_{m}^{L}\left(\lambda_{m, m}^{H}+\lambda_{f, m}^{H}\right) L_{m}+\left(\mu_{m}^{L}+\gamma_{m}^{L}\right) L_{m} \\
-\omega_{f}^{A} A_{f}+\delta_{f}^{L}\left(\lambda_{f, f}^{H}+\lambda_{m, f}^{H}\right) L_{f}+\left(\mu_{f}^{L}+\gamma_{f}^{L}\right) L_{f} \\
-\delta_{m}^{A}\left(\lambda_{m, m}^{H}+\lambda_{f, m}^{H}\right) A_{m}-\gamma_{m}^{Q} Q_{m}+\left(\mu_{m}^{P}+\omega_{m}^{P}\right) P_{m} \\
-\delta_{f}^{A}\left(\lambda_{f, f}^{H}+\lambda_{m, f}^{H}\right) A_{f}-\gamma_{f}^{Q} Q_{f}+\left(\mu_{f}^{P}+\omega_{f}^{P}\right) P_{f} \\
-\delta_{m}^{L}\left(\lambda_{m, m}^{H}+\lambda_{f, m}^{H}\right) L_{m}-\omega_{m}^{P} P_{m}+\left(\mu_{m}^{Q}+\gamma_{m}^{Q}\right) Q_{m} \\
-\delta_{f}^{L}\left(\lambda_{f, f}^{H}+\lambda_{m, f}^{H}\right) L_{f}-\omega_{f}^{P} P_{f}+\left(\mu_{f}^{Q}+\gamma_{f}^{Q}\right) Q_{f}
\end{array}\right) .
\end{aligned}
$$

Then, taking partial derivatives and evaluating at the endemic equilibrium, we have:

$$
F=\left(\begin{array}{ccccccccc}
\mathfrak{a}_{m} \frac{S_{m}^{*}}{N_{m}} & \mathfrak{b}_{m} \frac{S_{m}^{*}}{N_{m}} & 0 & 0 & \sigma_{m}^{V} \mathfrak{a}_{m} \frac{S_{m}^{*}}{N_{m}} & \sigma_{f}^{V} \mathfrak{b}_{m} \frac{S_{m}^{*}}{N_{m}} & 0 & 0 \\
\mathfrak{b}_{f} \frac{S_{f}^{*}}{N_{f}} & \mathfrak{a}_{f} \frac{S_{f}^{*}}{N_{f}} & 0 & 0 & \sigma_{m}^{V} \mathfrak{b}_{f} \frac{S_{f}^{*}}{N_{f}} & \sigma_{f}^{V} \mathfrak{a}_{f} \frac{S_{f}^{*}}{N_{f}} & 0 & 0 \\
0 & 0 & 0 & 0 & 0 & 0 & 0 & 0 \\
0 & 0 & 0 & 0 & 0 & 0 & 0 & 0 \\
\delta_{m}^{H} \mathfrak{a}_{m} \frac{H_{m}^{*}}{N_{m}} & \delta_{m}^{H} \mathfrak{b}_{m} \frac{H_{m}^{*}}{N_{m}} & 0 & 0 & \delta_{m}^{H} \sigma_{m}^{V} \mathfrak{a}_{m} \frac{H_{m}^{*}}{N_{m}} & \delta_{m}^{H} \sigma_{f}^{V} \mathfrak{b}_{m} \frac{H_{m}^{*}}{N_{m}} & 0 & 0 \\
\delta_{f}^{H} \mathfrak{b}_{f} \frac{H_{f}^{*}}{N_{f}} & \delta_{f}^{H} \mathfrak{a}_{f} \frac{H_{f}^{*}}{N_{f}} & 0 & 0 & \delta_{f}^{H} \sigma_{m}^{V} \mathfrak{b}_{f} \frac{H_{f}^{*}}{N_{f}} & \delta_{f}^{H} \sigma_{f}^{V} \mathfrak{a}_{f} \frac{H_{f}^{*}}{N_{f}} & 0 & 0 \\
0 & 0 & 0 & 0 & 0 & 0 & 0 & 0 \\
0 & 0 & 0 & 0 & 0 & 0 & 0 & 0
\end{array}\right) ; \quad V=\left(\begin{array}{cc}
\mathcal{A} & 0 \\
\mathcal{B} & \mathcal{C}
\end{array}\right),
$$


with

$$
\begin{gathered}
\mathcal{A}=\left(\begin{array}{cccc}
\mathfrak{c}_{m}+\mu_{m}^{A}+\omega_{m}^{A} & 0 & -\gamma_{m}^{L} & 0 \\
0 & \mathfrak{c}_{f}+\mu_{f}^{A}+\omega_{f}^{A} & 0 & -\gamma_{f}^{L} \\
-\omega_{m}^{A} & 0 & \mathfrak{d}_{m}+\mu_{m}^{L}+\gamma_{m}^{L} & 0 \\
0 & -\omega_{f}^{A} & 0 & \mathfrak{d}_{f}+\mu_{f}^{L}+\gamma_{f}^{L}
\end{array}\right), \\
\mathcal{B}=\left(\begin{array}{cccc}
-\mathfrak{c}_{m} & 0 & 0 & 0 \\
0 & -\mathfrak{c}_{f} & 0 & 0 \\
0 & 0 & -\mathfrak{d}_{m} & 0 \\
0 & 0 & 0 & -\mathfrak{d}_{f}
\end{array}\right), \\
\mathcal{C}=\left(\begin{array}{cccc}
\mu_{m}^{P}+\omega_{m}^{P} & 0 & -\gamma_{m}^{Q} & 0 \\
0 & \mu_{f}^{P}+\omega_{f}^{P} & 0 & -\gamma_{f}^{Q} \\
-\omega_{m}^{P} & 0 & \mu_{m}^{Q}+\gamma_{m}^{Q} & 0 \\
0 & -\omega_{f}^{P} & 0 & \mu_{f}^{Q}+\gamma_{f}^{Q}
\end{array}\right),
\end{gathered}
$$

where $\mathfrak{a}_{i}=q_{i} b_{i} \beta_{i, i}^{V}, \mathfrak{b}_{i}=\left(1-q_{j}\right) b_{j} \beta_{j, i}^{V}, \mathfrak{c}_{i}=\delta_{i}^{A} \mathfrak{A}_{i} \frac{H_{i}^{*}}{N_{i}}+\delta_{i}^{A} \mathfrak{B}_{i} \frac{H_{j}^{*}}{N_{i}}$ and $\mathfrak{d}_{i}=\delta_{i}^{L} \mathfrak{A}_{i} \frac{H_{i}^{*}}{N_{i}}+\delta_{i}^{L} \mathfrak{B}_{i} \frac{H_{j}^{*}}{N_{i}}$

Then, the invasion reproduction number for HSV-2 is given by:

$$
R_{H}^{V}=\rho\left(F V^{-1}\right)
$$




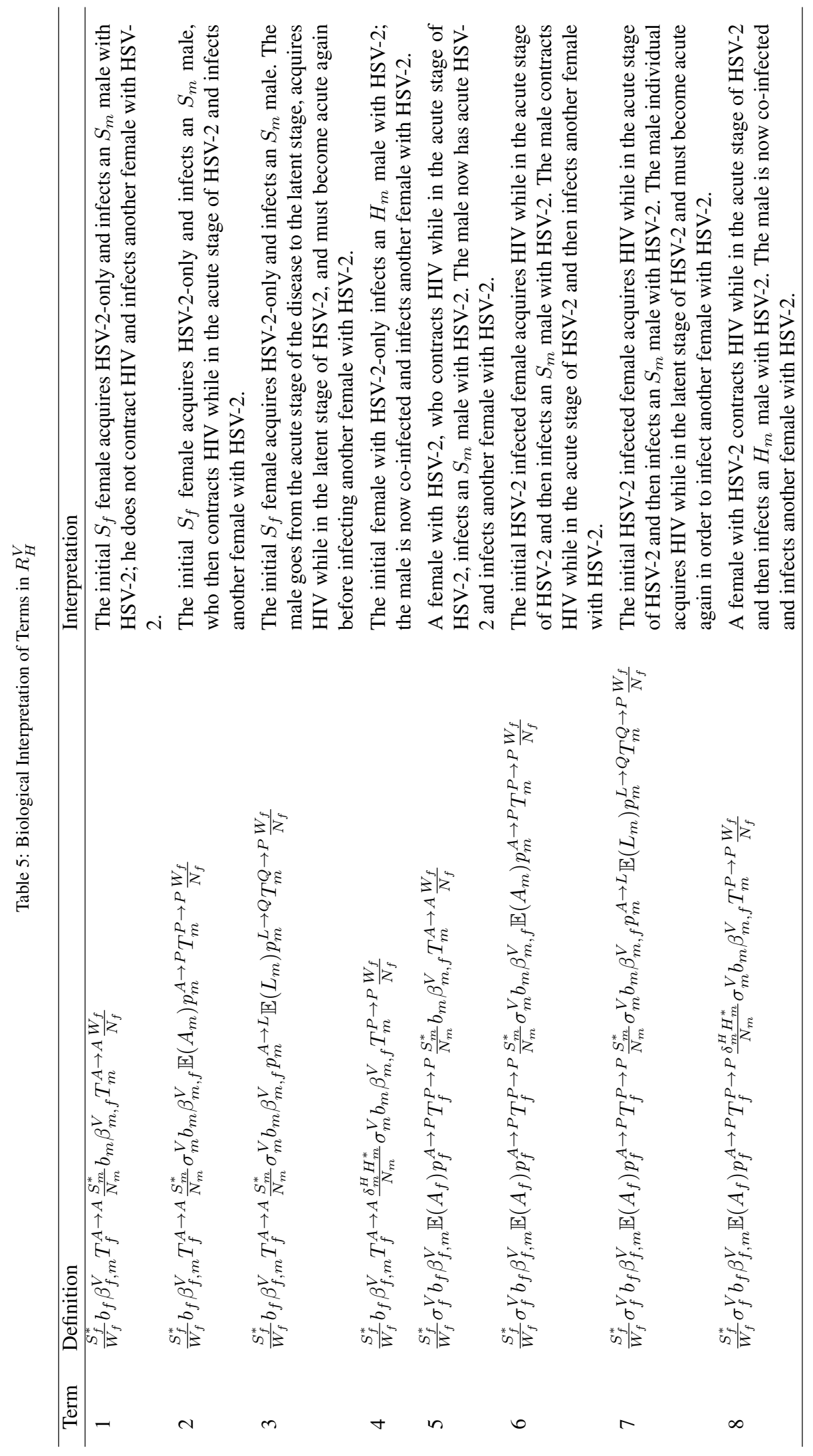




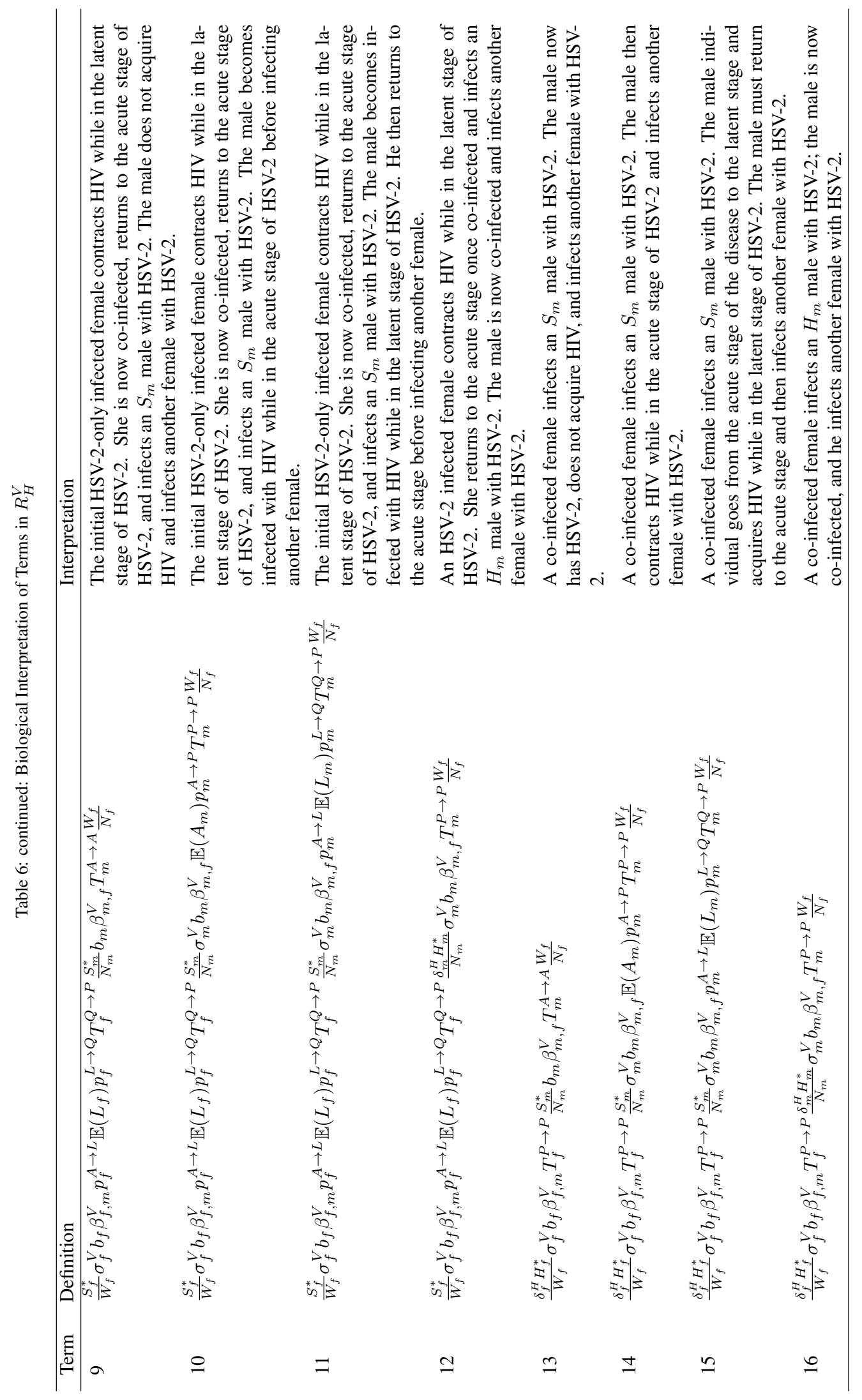


D.1 Interpretation of $\mathbb{E}\left(A_{i}\right), \mathbb{E}\left(L_{i}\right)$, and $T_{i}^{A \rightarrow A}$

The expressions $\mathbb{E}\left(A_{i}\right), \mathbb{E}\left(L_{i}\right)$, and $T_{i}^{A \rightarrow A}$ (see 4.13) can be derived as follows:

Let $h_{1}=\frac{\omega_{i}^{A}}{\mathcal{E}_{i}^{A}}$ be the probability that an individual with HSV-2-only progresses from the acute stage to the latent stage of HSV-2 before acquiring HIV or exiting the system. Similarly, let $h_{2}=\frac{\gamma_{i}^{L}}{\mathcal{E}_{i}^{L}}$ be the probability that an individual with HSV-2-only in the latent stage returns to the acute stage before acquiring HIV or exiting the system. Then the expected number of times that an HSV-2 infected individual in the acute stage of the disease, who does not acquire HIV, remains in the acute stage is:

$$
\begin{aligned}
\mathbb{E}\left(A_{i}\right) & =\sum_{k=0}^{\infty}\left(h_{1} h_{2}\right)^{k} \\
& =\frac{1}{1-h_{1} h_{2}} \\
& =\frac{\mathcal{E}_{i}^{A} \mathcal{E}_{i}^{L}}{\mathcal{E}_{i}^{A} \mathcal{E}_{i}^{L}-\omega_{i}^{A} \gamma_{i}^{L}} .
\end{aligned}
$$

Similarly, the expected number of times that an HSV-2 infected individual in the latent stage of the disease, who does not acquire HIV, remains in the latent stage is:

$$
\begin{aligned}
\mathbb{E}\left(L_{i}\right) & =\sum_{k=0}^{\infty}\left(h_{2} h_{1}\right)^{k} \\
& =\frac{1}{1-h_{2} h_{1}} \\
& =\frac{\mathcal{E}_{i}^{A} \mathcal{E}_{i}^{L}}{\mathcal{E}_{i}^{A} \mathcal{E}_{i}^{L}-\omega_{i}^{A} \gamma_{i}^{L}}
\end{aligned}
$$

Hence, as in 4.13, $\mathbb{E}\left(A_{i}\right)=\mathbb{E}\left(L_{i}\right)=\frac{\mathcal{E}_{i}^{A} \mathcal{E}_{i}^{L}}{\mathcal{E}_{i}^{A} \mathcal{E}_{i}^{L}-\omega_{i}^{A} \gamma_{i}^{L}}$.

Now, the average time spent in the $A_{i}$ class each time is $\frac{1}{\mathcal{E}_{i}^{A}}$. Hence, as in 4.13, the overall time spent in the $A_{i}$ class, if the individual does not acquire HIV, is:

$$
T_{i}^{A_{i} \rightarrow A_{i}}=\frac{1}{\mathcal{E}_{i}^{A}} \frac{\mathcal{E}_{i}^{A} \mathcal{E}_{i}^{L}}{\mathcal{E}_{i}^{A} \mathcal{E}_{i}^{L}-\omega_{i}^{A} \gamma_{i}^{L}}=\frac{\mathcal{E}_{i}^{L}}{\mathcal{E}_{i}^{A} \mathcal{E}_{i}^{L}-\omega_{i}^{A} \gamma_{i}^{L}} .
$$

